\title{
Studienfachwahl als Spezialfall der Ausbildungsentscheidung und Berufswahl
}

\author{
Rolf Becker $\cdot$ Sigrid Haunberger $\cdot$ Frank Schubert
}

Angenommen: 9. September 2009 / Online veröffentlicht: 9. Oktober 2009

(C) Institut für Arbeitsmarkt- und Berufsforschung 2009

Zusammenfassung Vorliegende Studien belegen eine persistente horizontale Ungleichheit von Studienfachwahlen nach sozialer Herkunft. Im vorliegenden Beitrag wird der Frage nachgegangen, warum ein Zusammenhang zwischen sozialer Herkunft und Studienfachwahl besteht. Auf der Datenbasis der sächsischen Abiturientenbefragung seit 2000 kann die Entscheidungsfindung der Abiturientinnen und Abiturienten zu ihren nachschulischen Bildungs- und Berufswegen etwa drei Monate vor dem Abitur modelliert werden. Durch die empirischen Analysen kann nachgewiesen werden, dass die Studienfachwahl in Abhängigkeit von der Schichtzugehörigkeit das Ergebnis von Entscheidungen ist, die durch das Motiv des Statuserhalts, den erwarteten Kosten für bestimmte Ausbildungen, den individuellen Leistungspotenzialen, dem erwarteten Studienerfolg und der soziokulturellen Distanz zur tertiären Bildung strukturiert werden. Anhand dieser Determinanten der Studienfachwahl kann der Effekt sozialer Herkunft und damit - im Aggregat gesehen - die persistente soziale Ungleichheit von Studienfachwahlen nach sozialer Herkunft erklärt werden.

\section{The choice of field of study as a special case of decision on tertiary training and occupation}

Abstract Recent studies provide empirical evidence on persistence of horizontal inequality of field of study depend-

R. Becker $(-$ S. Haunberger $\cdot$ F. Schubert

Universität Bern, Institut für Erziehungswissenschaft, Abteilung

Bildungssoziologie,

Muesmattstrasse 27, 3012 Bern, Schweiz

E-Mail: rolf.becker@edu.unibe.ch

Tel.: +41-31-6315351

Fax: +41-31-6315352 ing on eligible individuals' social origin. In this article we explore the question why there is a correlation between social origin and choice of field of study. Using data from the Saxon survey of high school graduate candidates, we model the decision process of high school students regarding postsecondary education about three month prior to graduation. Our empirical results prove that the effect of social origin on the choice of field of study is the result of an individual decision structured by status maintenance motive, by expected costs for the different types of higher education and training, by individual ability level, by expected success probabilities as well as by the socio-cultural distance to tertiary education. These determinants of the choice of field of study explains the effect of social origin and, therefore for the aggregate, the persistent social inequality of opportunities in the access to field of study.

\section{Einleitung}

Viele empirische Studien in der Hochschulforschung belegen einen systematischen Zusammenhang zwischen sozialer Herkunft und Ausbildungsentscheidung von Abiturienten (Müller u. Jacob 2008; Becker u. Hecken 2007; Mayer et al. 2007; Arum et al. 2007). Demnach entscheiden sich Absolventen aus den höheren Sozialschichten relativ häufig für ein Hochschulstudium, während Arbeiterkinder wiederum eher von Universitäten abgelenkt werden (Becker u. Hecken 2008; Müller u. Pollak 2007). Diese soziale Selektivität des Hochschulzugangs wird darüber hinaus auch für die Auswahl eines bestimmten Studienfachs nachgewiesen (Reimer u. Pollak 2009; Heine u. Willich 2006; Van de Werfhorst et al. 2001; Davies u. Guppy 1997). So präferieren studienbereite Akademikerkinder vor allem Studienrichtungen wie etwa Medizin oder Jura, 
während Lehramtsstudiengänge oder Studienfächer wie Pädagogik oder Elektrotechnik besonders für Arbeiterkinder interessant zu sein scheinen (Reimer u. Pollak 2005).

Um den Zusammenhang von sozialer Herkunft und Studienfach zu erklären, wird zumeist auf die Arbeiten von Bourdieu (1983, 1984) oder von Bourdieu u. Passeron (1978) über die intergenerationale Transmission der elterlichen Ressourcen - insbesondere des kulturellen Kapitals - und der Reproduktion des Studienfachs zurückgegriffen (Schölling 2005). Sich hauptsächlich an Bourdieu anlehnend, legen Van de Werfhorst et al. (2001) in einer Studie für die Niederlande drei Erklärungen für den Zusammenhang von familiärem Hintergrund und der Studienfachwahl vor. Erstens wird von einer direkten intergenerationalen Transmission der Präferenzen und Aspirationen von den Eltern auf die Kinder ausgegangen, sodass - durch die Erziehung im Elternhaus und die Selbstselektion im Bildungsverlauf bedingt - die Kinder die selbe Ausbildung durchlaufen und das gleiche Studienfach studieren. Zweitens beeinflusst das elterliche kulturelle und ökonomische Kapital die Studienfachwahl: In der Regel wird über die Vermittlung von Lebensstilen und Werten in der Erziehung die Reproduktion der ökonomischen Elite über ein wirtschaftswissenschaftliches Studium sowie die Reproduktion der kulturellen Elite über ein kulturwissenschaftliches Studium sichergestellt. Drittens korrelieren Studienfach und soziale Herkunft miteinander, weil - entsprechend der "Status Position Theory“ von Boudon (1974) - die Absolventen aus unterschiedlichen Sozialschichten in ihren Wahrnehmungen und Evaluationen von Kosten und Nutzen bestimmter Studienfächer differieren. So neigen Arbeiter- und Mittelschichten zu einem technischen und ökonomischen Studienfach, weil diese - gemessen an den ökonomischen Ressourcen und dem sozioökonomischen Status des Elternhauses - einen Statusaufstieg ermöglichen.

In eine ähnliche Richtung gehen Erklärungsversuche, welche - ebenfalls in Anlehnung an Bourdieu (1984) den Zusammenhang von sozialer Herkunft und Studienfach auf eine Passung von Herkunfts- und Studienfachkultur zurückführen (Windolf 1990). Das Studieren eines bestimmten Studienfachs ist Ausdruck von zwischen Sozialschichten differierenden alltagsästhetischen Präferenzen (Einstellungen und Werte) oder dem „Anlagesinn für die ,richtigen “ Ausbildungsgänge“ (d. h. Übereinstimmung des herkunftsbedingten Selbstbildes, der kulturellen Praxen und der Wissensbestände mit der sozial zugeschriebenen Fachkultur) (Georg 2005, S. 62). Folglich wird dem Studienfach entsprochen, das am ehesten dem Streben nach Distinktion sowie dem herkunftsbedingten „Habitus“ und kognitiven Stil entspricht. Die Studienfachwahl reflektiert demnach die Affinität für einen fachspezifischen Lebensstil und eine bestimmte Studienfachkultur. Das Studium eines bestimmten Faches geht mit der Wahl einer bestimmten
„Lebensform“ einher, die mit internalisierten Werten und dem herkunftsbezogenen Selbstbild kompatibel ist (Windolf 1990). Studierende aus höheren Sozialschichten verfügen über eine höhere Passung von Herkunfts- und Studienfachkultur und besitzen - so Georg (2005, S. 62) - somit einen Wettbewerbsvorteil gegenüber Studierenden aus unteren Sozialschichten. Wenn jedoch Studenten aus höheren Sozialschichten über den Zugang zu Hochkultur über mehr „kulturelles Kapital“ verfügen, dann stellt sich allerdings die Frage, wie Kenntnisse und Vertrautheit im Bereich der Hochkultur nahtlos mit dem Studium von Medizin oder Jura einhergehen oder wie die soziale Reproduktion der sozialen Ungleichheit durch die Hierarchisierung der Studienfächer vonstatten geht.

Selbst wenn man davon ausgeht, dass der intergenerationale Statuserhalt das dominante Ziel der Auswahl eines bestimmten Studienfachs ist, dann bleiben bei der Anwendung des Ansatzes von Bourdieu (1984) die Mechanismen der Studienfachwahl selbst im Dunkeln (vgl. Hedström u. Swedberg 1996). ${ }^{1}$ Kultureller „Habitus“ und andere im Erziehungs- und Sozialisationsprozess übernommene „Gewohnheiten“ setzen auch für die Studienfachwahl einen vollständig sozialisierten ,homo academicus“ voraus, dessen Ausbildungsentscheidung auf traditionalem Handeln basiert. Auf welche Traditionen sollen dann Bildungsaufsteiger zurückgreifen? Ein Ausweg aus diesem theoretischen Dilemma wird in der Analyse von Neigungen und Motivationen gesucht, die leitend für die Auswahl einer bestimmten Studienrichtung sein sollen (Heublein u. Sommer 2002; Lischka u. Wolter 2001). Neigungen und Motivation (wie etwa Interesse an Wissenserwerb oder Selbstverwirklichung, Bildung an sich oder fachwissenschaftliches Interesse) sind sicherlich notwendige, aber keine hinreichenden Gründe für eine Ausbildungs- und Berufswahl (Böttcher et al. 1988, S. 12). Unklar bleibt bei den vorliegenden Studien, wie Neigungen, Motivation und darauffolgende Studienfachwahlen mit der sozialen Herkunft zusammenhängen. Abgesehen von dieser ungeklärten

\footnotetext{
${ }^{1}$ In der Terminologie von Max Weber verbleibt die von Bourdieu geteilte Sichtweise über die Studienfachwahl allerdings bei der Rekonstruktion eines „,sinnhaft adäquaten“ Verhaltens - ein „zusammenhängend ablaufendes Verhalten in dem Grade (...), als die Beziehung seiner Bestandteile von uns nach den durchschnittlichen Denk- und Gefühlsgewohnheiten als typischer (wir pflegen zu sagen: ,richtiger") Sinnzusammenhang bejaht wird“ (Weber 1980, S. 5). Das entspricht etwa dem „Anlagesinn für die ,richtigen“ Ausbildungsgänge“ (Georg 2005). Die Kausaladäquanz bleibt jedoch unberücksichtigt, sodass - in der Logik Max Webers - die Theorie von Bourdieu nicht in der Lage ist, Richtung und Wahrscheinlichkeit einer bestimmten Studienfachwahl vorherzusagen. Da sie keine Mechanismen benennt, wie sich Motive in Handeln umsetzen, also wie Sinn- und Kausaladäquanz einhergehen, kann sie dieses Verhalten auch nicht kausal erklären, weil keine direkte empirisch überprüfbare Verbindung zwischen der zu erklärenden Verteilung von Studienfachwahlen und der gegebenen Sozialstruktur der Studienberechtigten hergestellt wird. Damit ist der Erklärungsversuch von und nach Bourdieu unvollständig.
} 
Frage weisen - von wenigen Ausnahmen abgesehen (Heine u. Willich 2006, S. 22-24; Becker 2000b) - die empirischen Untersuchungen dazu das gravierende methodische Problem auf, solche Neigungen und (intrinsische wie extrinsische) Motivationen nur für Studierende nach ihrem Studienbeginn zu analysieren (Asmussen 2006). Sowohl in Bezug auf die herkunftsbedingte Entscheidung für eine bestimmte Ausbildung als auch für die Wahl eines bestimmten Studienfachs in Abhängigkeit der sozialen Herkunft fehlt eine Referenzkategorie, die plausibel macht, warum sich bei Kontrolle von Neigungen und Motivationen bestimmte Sozialschichten oder zumindest einige Studienberechtigte aus mehr oder weniger bildungsfernen Schichten weder für ein Hochschulstudium noch für eine bestimmte Studienrichtung entscheiden (vgl. Böttcher et al. 1988). Vielmehr ist davon auszugehen, dass die Befunde solcher Untersuchungen wegen sozial selektiver Stichproben oder wegen sozialer Erwünschtheit oder wegen Rationalisierung von Gründen für eine bereits getroffene Ausbildungsentscheidung erheblich verzerrt sind. ${ }^{2}$

Vor dem Hintergrund dieser theoretischen und methodischen Probleme gehen wir folgender Fragestellung nach: Warum gibt es einen Zusammenhang zwischen sozialer Herkunft und Studienfachwahl? In der Rekonstruktion einer erklärenden Antwort wird wegen der aufgezeigten Defizite der bislang angewandten Erklärungsversuche nach Bourdieu eine alternative Theorie gewählt: Aus entscheidungsund handlungstheoretischer Sicht wird davon ausgegangen, dass die Studienfachwahl ein Spezialfall der Ausbildungsentscheidung und eine vorweggenommene Berufswahl ist, die systematisch von der sozialen Herkunft der Studienberechtigten und ihres vorhergehenden Bildungsverlaufs abhängt.

Es ist ein Ziel der vorliegenden Untersuchung, die Herkunftsabhängigkeit von Studienfachwahlen mit einem entscheidungs- und handlungstheoretischen Ansatz - der Theorie subjektiv erwarteten Nutzens - zu erklären. Es soll empirisch nachgewiesen werden, dass die soziale Herkunft als erklärende Variable durch die individuelle Abwägung von Kosten und Nutzen von Studienfächern, die wiederum von der sozialen Situation und den verfügbaren Ressourcen abhängt, erklärt werden kann. Ein weiteres Ziel ist es, im Sinne kumulativer empirischer Forschung - anschließend an die Arbeit von Becker u. Hecken (2007, 2009b) - einen weiteren Beitrag zur Erklärung der schichtspezifischen Reproduktion des Hochschulstudiums und des Studienfaches

\footnotetext{
${ }^{2}$ Beispielsweise erwartet man von einem Medizinstudenten, dass er dieses Fach aus Nächstenliebe studiert, um anderen Menschen zu helfen und nicht um sich selbst mit hohem Status und Einkommen zu versorgen. Diese allgemein geteilte Erwartung und auch das Selbstbild, das sich auch die Medizin selbst gibt, dürfte die Neigung zu sozialer Erwünschtheit bei Fragen nach den Gründen für ein Medizinstudium verstärken.
}

und damit auch zur Erklärung persistenter Ungleichheiten beim Zugang zur tertiären Bildung zu liefern.

Um dieses Erklärungsmodell und zentrale Hypothesen empirisch zu überprüfen, wird auf Daten der sächsischen Abiturientenbefragung zurückgegriffen (vgl. Wolter et al. 2006). Abgesehen davon, dass die Entscheidung der Abiturienten detailliert rekonstruiert werden kann, liegt ein besonderer Vorzug dieser Datenbasis darin, dass die Rationalisierung von Ausbildungsentscheidungen deswegen ausgeschlossen ist, weil die Abiturientinnen und Abiturienten bereits drei Monate vor dem Abitur zu ihren nachschulischen Bildungs- und Berufswegen befragt wurden. Ebenso von Vorteil ist, dass bei dieser Befragung das Problem einer fehlenden Referenzkategorie zu den Studierenden nicht gegeben ist.

Im zweiten Kapitel des Beitrags wird die Theorie des subjektiv erwarteten Nutzens für eine Ausbildungsentscheidung zwischen Berufsausbildung und Studium - ein strukturell-individualistischer Erklärungsansatz, der sich empirisch bewährt hat - so erweitert, dass die individuelle Entscheidung zwischen verschiedenen Studienfächern in Konkurrenz zu anderen Alternativen modelliert wird. Aus diesem werterwartungstheoretischen Modell werden empirisch überprüfbare Hypothesen abgeleitet. Das dritte Kapitel umfasst die Beschreibung der Datenbasis, der Variablen und des statistischen Verfahrens. Die Darstellung und Interpretation der empirischen Befunde erfolgt im vierten Kapitel. Im fünften Kapitel werden die Ergebnisse zusammengefasst und im Hinblick auf den Forschungsstand und die zukünftige Theorie- und Modellbildung diskutiert.

\section{Theoretischer Hintergrund}

\subsection{Grundlagen}

Nach Hillmert u. Jacob (2003) ist die Entscheidung, nach dem Abitur ein Studium oder eine berufliche Ausbildung zu absolvieren, eine sinnhafte Entscheidung unter erheblicher Unsicherheit und daher eine idealtypische Anwendung für entscheidungs- und handlungstheoretische Modelle, die zur Familie aufgeklärter Rational-Choice-Theorien zählen. Aus entscheidungs- und handlungstheoretischer Sicht ist auch für Studienberechtigte (und aus Sicht ihres Elternhauses) sowohl die Entscheidung für eine bestimmte Berufsausbildung (etwa Ausbildung zum Anlagenmechaniker, Maurer oder Zimmerer) oder für eine bestimmte Studienrichtung (etwa Jura, Medizin oder Sozialwissenschaften) als ein Spezialfall für die Entscheidung zwischen Studium oder Berufsausbildung anzusehen (Becker u. Hecken 2007). In methodischer Hinsicht bedeutet das, dass die unbeobachtete Heterogenität für die Entscheidungsalternativen einer nachschulischen Ausbildung berücksichtigt wird. Diese 
Spezifikation ist zunächst aus Sicht der Theorie sozialer Produktionsfunktionen (Lindenberg 1989) naheliegend (zur Kritik: Opp u. Friedrichs 1996). Demnach reicht es beispielsweise für die obere Dienstklasse, insbesondere Familien mit einer akademischen oder professionellen Tradition, nicht aus, dass ihre Kinder irgendein beliebiges Studienfach studieren, damit in der Generationenfolge die Statuslage, gemessen an Prestige, Einkommen oder Klassenlage, erhalten werden kann und gleichzeitig - im Sinne der „Social Position Theory“ von Boudon (1974) ein Statusverlust vermieden werden kann (Reimer u. Pollak 2009, S. 3; Davies u. Guppy 1997, S. 1419; Hansen 1997, S. 308). So heben Reimer u. Pollak (2009) für das deutsche binäre System der tertiären Bildung hervor, dass in Bezug auf den Statuserhalt der Studienfachwahl deswegen eine besondere Bedeutung zukommt, weil hinsichtlich der Reputation und des Prestiges die Unterschiede zwischen den Universitäten deutlich geringer sind als in diversifizierten Systemen höherer Bildung mit Eliteinstitutionen (z. B. Großbritannien, Frankreich oder USA).

Hinzuzufügen ist, dass mit der Studienfachwahl auch angestrebte Zwischengüter einhergehen wie etwa Chancen, in eine Berufstätigkeit zu gelangen, die eine Optimierung von Einkommen, gesellschaftlichem Ansehen und vor allem Klassenlage wahrscheinlich macht. Auf diesem Weg soll der sich daraus ergebende Nutzen infolge des Zugangs zu einer möglichst privilegierten Klassenlage oder Positionierung in der gesellschaftlichen Schichtung der intergenerationale Statuserhalt sichergestellt werden (Müller 1994). Daher konzentrieren sich die oberen Dienstklassen in Deutschland darauf, dass ihre Kinder die lukrativen und prestigeträchtigen Studienfächer mit entsprechenden Einkommens- und Statuschancen studieren. ${ }^{3}$ Vor allem für Mediziner oder Juristen ist zwingend geboten, sei es, um eine Familientradition fortzusetzen (Multrus 2006) oder sei es, um eine Praxis samt Einrichtung weiterzugeben, dass ihre Kinder (und Kindeskinder) ebenfalls Medizin bzw. Rechtswissenschaft studieren. Für selbstständige Landwirte oder Handwerker hingegen ist es nicht notwendig, dass ihre Kinder studieren, um den bislang erreichten Status zu erhalten (Hansen 1997 S. 318; Müller 1975). Vielmehr sind sie daran interessiert, dass ihre Kinder das gleiche Handwerk erlernen, um den Betrieb übernehmen zu können. Insofern gelten bei der Studienfachwahl identische Mechanismen der subjektiven Wahrnehmung und Bewertung

\footnotetext{
${ }^{3}$ Sie entscheiden sich dann auch eher für die traditionellen Universitäten statt für Fachhochschulen oder für das Angebot der nichttertiären Berufsausbildung (Müller et al. 2002). Des Weiteren kann im Sinne der „Maximally Maintained Inequality"-Hypothese argumentiert werden, dass im Zuge der Bildungsexpansion die höheren Sozialschichten in zunehmendem Maße sozial exklusive Studienfächer wählen, um ihre relativen Privilegien abzusichern (Lucas 2001). Für Deutschland konnte diese Annahme empirisch nicht bestätigt werden (Reimer u. Pollak 2009).
}

der Alternativen zwischen den Studienfächern im Kontrast zu nichttertiären Ausbildungen wie bei der Entscheidung zwischen Hochschulstudium und Berufsausbildung (vgl. Becker u. Hecken 2007, 2009b).

Einige Restriktionen, sich zwecks Statuserhalt für eine spezifische Ausbildung zu einem bestimmten Beruf entscheiden zu können, ergeben sich nicht nur aus dem institutionellen Bildungsangebot selbst, sondern auch aus früher getroffenen Bildungsentscheidungen (Breen u. Jonsson 2000; Becker u. Hecken 2009a). Personen, die sich - aus welchen Gründen auch immer - für eine Schulausbildung entschieden haben oder entscheiden mussten, die nicht zur Studienberechtigung führte, verfügen über ein anderes Set von Ausbildungsalternativen als Personen mit einer Hochschulreife (Wolter et al. 2006; Opp u. Friedrichs 2002). Neben dieser Kontingenz des Bildungsverlaufs und der endogenen Pfadabhängigkeit von Bildungschancen ergeben sich weitere Restriktionen aus der Soziallage selbst, die den vorherigen Bildungsverlauf mitbestimmt haben (Müller u. Karle 1993). Individuelle Leistungspotenziale, ökonomische Ressourcen für Ausbildungsinvestitionen und soziokulturelle Distanzen zum System höherer Bildung sind solche. Im Aggregat ergibt sich aus diesen individuellen, zwischen den einzelnen Sozialschichten differierenden Studienfach- bzw. Berufsentscheidungen die „Sozialstruktur“ der studierten Fächer und erlernten Berufen.

\subsection{Spezifikation des erklärenden Modells}

Um die individuelle Studienfachwahl im Kontrast zu anderen Ausbildungen zu modellieren, wird die von Esser (1999, S. 265-275) in Anlehnung an Boudon (1974) sowie Breen u. Goldthorpe (1997) vorgeschlagene Theorie der Bildungsentscheidung erweitert (vgl. Becker u. Hecken 2009b). Für studienberechtigte Personen ergeben sich - abgesehen vom unmittelbaren Übergang in den Arbeitsmarkt oder in einen anderen Zustand - zwei Alternativen, den Bildungsverlauf fortzusetzen: (nichttertiäre) Berufsausbildung $\left(B_{i}\right)$ oder Studienfach $\left(S_{i}\right) .{ }^{4}$ Die Entscheidung zugunsten eines Studienfachs, die mehr oder weniger zeitlich synchron mit der Entscheidung zugunsten eines Hochschulstudiums und in selteneren Fällen sequenziell (zuerst Studium, dann Studienfach oder noch seltener: zuerst Studienfach, dann Uni-

\footnotetext{
${ }^{4}$ Die Konsequenzen der jeweiligen Entscheidung ergeben die Bildungsrendite $U_{i}$. Indirekte und direkte Kosten in Höhe $-C_{i}$, die bei einem Studium eines Studienfachs $S_{i}$ entstehen, und der drohende Statusverlust in Höhe von $-S V_{i}$, der bei einer den sozialen Status nicht garantierenden Studienfachwahl auftritt, sind weitere Bestandteile des Kosten-Nutzen-Kalküls der evaluierenden und entscheidenden Personen. Schließlich bilden die beiden Erwartungswerte $p_{i}$ und $c_{i}$ jeweils die Wahrscheinlichkeit für den Bildungserfolg und die Erwartung eines Statusverlustes bei einem Verzicht auf das Studieren eines bestimmten Faches.
} 
versität oder Fachhochschule) fällt (vgl. Becker 2000b), wird dann getroffen, wenn der zu erwartende Nutzen für eine bestimmte Studienrichtung $i$ größer ist als für andere Bildungsalternativen $i \operatorname{SEU}\left(S_{i}\right)=p_{i} U_{i}+\left(1-p_{i}\right)$ $c_{i}\left(-S V_{i}\right)-C_{i}>\operatorname{SEU}\left(B_{i}\right)=c_{i}\left(-S V_{i}\right) .^{5}$ Wenn für ein bestimmtes Studienfach die Bildungsmotivation $(U+c S V)$ das Investitionsrisiko $(C / p)$ für dieses Studienfach dominiert sowie für alle anderen Alternativen geringere Bildungsmotivationen oder höhere Investitionsrisiken vorliegen, dann entscheidet sich eine Person schließlich mit großer Wahrscheinlichkeit für dieses eine Studienfach.

Die Orientierung für die Auswahl eines Studienfachs oder für die Auswahl einer anderen Berufsausbildung muss - so das Modell der Frame-Selektion (Kroneberg 2005) nicht zwangsläufig rein zweckrational sein und einem zweckrationalen Handeln vorausgehen, bei denen Vor- und Nachteile einzelner Ausbildungen reflektiert kalkulierend abgewogen werden (Esser 2001; Meulemann 2001, S. 73). ${ }^{6}$ Der Studienfachwahl können auch wertrationale Orientierungen zugrunde liegen (Kroneberg 2007). Ein Beispiel wäre der Glaube an die Berufung zum Lehrer oder zum Politiker, der die Entscheidung für ein Lehramtsstudium bzw. Studium der Politologie oder Rechtswissenschaft sehr wahrscheinlich macht. In manchen Fällen kann dem Handeln eine traditionale Orientierung zugrunde liegen, wenn beispielsweise eine Familientradition besteht, infolge der eine Rechtsanwaltkanzlei, eine Zahnarztpraxis oder eine Zimmermannswerkstatt von Generation zu Generation vererbt wird. In diesem Fall werden zumeist ,automatischspontane Entscheidungen“ zu Gunsten einer Ausbildung gefällt, die zu einem bestimmten Beruf oder Berufsfeld führen, sodass die Fortsetzung dieser Familientradition garantiert ist. Solche routinierten Ausbildungsentscheidungen

\footnotetext{
${ }^{5}$ In theoretischer Hinsicht kann eine synchrone Entscheidung insofern plausibel sein, dass sich Abiturienten von vornherein für ein Studienfach entscheiden. Solch eine Entscheidung denkt die Entscheidung für ein Studium an einer Hochschule implizit mit. Für bestimmte Studienfächer wie eben Medizin oder Jura fällt alleine aus institutionellen Gründen die Entscheidung zu Gunsten eines Universitätsstudiums aus. Synchrone Entscheidung dürfte vor allem für Akademiker(kinder) wahrscheinlich sein. Gerade für diese Sozialschichten müsste empirisch festzustellen sein, dass sie einerseits im Vergleich zu anderen Sozialschichten sehr früh eine Studienentscheidung treffen. Zum Anderen ist für den Fall einer bereits getroffenen Studienentscheidung anzunehmen, dass sich vor allem Angehörige der Klasse der Akademiker und Professionellen für ein bestimmtes Studienfach entschieden haben.

${ }^{6}$ Die Rationalität dieser Ausbildungsentscheidungen liegt darin, dass Studienberechtigte - ausgehend von der Positionierung ihres Elternhauses im Schichtungsgefüge einer Gesellschaft - aus dem für sie bekannten und zur Verfügung stehenden Set von Handlungen diejenige Alternative auswählen, die sie als die optimale ansehen, um ein übergeordnetes Ziel - im vorliegenden Fall: Statuserhalt - zu erreichen. Diese Entscheidung ist eingebettet in eine lebenslaufbezogene Strategie des Elternhauses, den Status zu erhalten und an die nächste Generation weiterzugeben (Meulemann 1985). In modernen Gesellschaften sind langfristige Investitionen in Bildung und Ausbildung der nachwachsenden Generation eine Erfolg versprechende Strategie für den Statuserhalt.
}

dürften für Akademiker typisch sein, da sie über dominante kognitive Rahmen (,frames“) zu Sinn und Zweck von akademischer Ausbildung und bestimmten Disziplinen ebenso verfügen wie über eindeutiges wert- oder traditionsbasiertes Wissen über in dieser Entscheidungssituation gebotene oder geforderte Handlungsabläufe (,scripts“) sowie schließlich über für die Entscheidungssituation angemessene Handlungsroutinen (,habits“) (Esser 2001). Daher entscheiden sie sich unhinterfragt aus Gewohnheit oder wegen unbedingt mit Überzeugung vertretenen Werthaltungen für ein Universitätsstudium und für das Studienfach, welches ohnehin - wie auch von Bourdieu (1984) angenommen - in der Tradition der Familie liegt. Erst wenn die entsprechenden Voraussetzungen nicht mehr erfüllt sind, wenn beispielsweise die schulischen Leistungen nicht mehr stimmen oder gar das Abitur gefährdet scheint, wird statt eines automatisch-spontanen Entscheidens ein reflektiertes Kalkulieren über Kosten und Nutzen von Alternativen einsetzen.

\subsection{Erklärung der schichtspezifisch unterschiedlichen Ausbildungsentscheidung}

Wie kann anhand dieses theoretischen Modells die zwischen Sozialschichten variierende Entscheidung für ein bestimmtes Studienfach erklärt werden? Zunächst ist es sinnvoll, zwischen primären und sekundären Herkunftseffekten zu unterscheiden und bei gegebenen institutionellen Opportunitäten und Restriktionen ihr Zusammenspiel für die Studienfachwahl zu rekonstruieren (Boudon 1974). Auch wenn es sich bei Abiturienten sozial und leistungsbezogen um eine selektive Gruppe handelt, die bislang alle Übergänge im Bildungssystem ,,überlebt“ hat (Davies u. Guppy 1997; Blossfeld 1993), sind primäre Herkunftseffekte - d. h. Auswirkungen der sozialen Herkunft auf die Schulleistung, Leistungspotenziale, die darauf aufbauenden Studienchancen und Auswahl eines bestimmten Studienfachs - zu erwarten (Breen u. Goldthorpe 1997; Becker 2009). Weil Abiturienten aus den höheren Sozialschichten relativ bessere Schulnoten und eine höhere Erwartung aufweisen, ein Studium erfolgreich bewältigen zu können, werden sie sich in einem geringeren Maße von den Anforderungen bestimmter, in der Regel auch prestigeträchtiger Studienfächer (wie etwa Medizin, Jura etc.) abschrecken lassen. Abiturienten aus unteren Sozialschichten werden aufgrund ihrer geringeren Erfolgserwartungen eher Studienfächer wählen, die als weniger anspruchsvoll gelten. Im Unterschied zu diesen Abiturienten werden sozial privilegierte Abiturienten auch eher die Hürden des Numerus clausus für einige Studienfächer (wie etwa Medizin oder Psychologie) nehmen und nicht von den Universitäten wegen unzureichender Abschlussnoten abgewiesen (Böttcher et al. 1988, S. 13). 
Aufgrund primärer Herkunftseffekte werden sich im Vergleich zu Abiturienten aus den unteren und mittleren Sozialschichten vor allem Abiturienten aus der Oberschicht für die prestigeträchtigen und qualifikatorisch voraussetzungsund anspruchsvollen Studienfächer (einschließlich der NC-Fächer) entscheiden (können). Weil andere Fächer wie etwa Betriebswirtschaftslehre, Erziehungs- oder Sozialwissenschaften das Image eines wenig anspruchsvollen Faches zugeschrieben wird, stellen sie für Bildungsaufsteiger aus den Arbeiterklassen geringe Hürden dar. Weil sie glauben, diese Studienrichtungen auch erfolgreich studieren $\mathrm{zu}$ können, werden sie sich eher für diese entscheiden als Abiturienten aus den höheren Sozialschichten.

Die sekundären Herkunftseffekte reflektieren bei der vorliegenden Fragestellung die Auswirkungen der sozialen Herkunft auf die individuelle Entscheidung für ein bestimmtes Studienfach. Zunächst werden aufgrund des Statuserhaltungsmotivs die mittleren und vor allem die höheren Sozialschichten (sprich: die vornehmlich aus Akademikern, Professionellen sowie Beamten und Angestellten in Leitungspositionen bestehende obere Dienstklasse) daran interessiert sein, dass ihre Kinder solche Studienfächer wählen, die den Zugang zu prestigeträchtigen Berufen und Professionen mit hohen Bildungsrenditen ermöglichen, welche wiederum geeignet sind, Statusverluste zu vermeiden. Insbesondere Kinder von Professionellen (etwa Ärzte und Juristen) werden strukturell ,gezwungen“ sein, sich für eine Studienrichtung zu entscheiden, die Statusverluste unwahrscheinlich machen. Dieses Faktum würde für die obere Dienstklasse die Neigung für Studienfachentscheidungen erklären, die zu einer hochgradigen Berufsvererbung und Reproduktion des bislang erreichten Status führen.

Eine gewisse empirische Evidenz für diese Annahme der Nutzenmaximierung lässt sich aus der von Wegener (1988) vorgeschlagenen Magnitude-Prestigeskala für die Klassifikation der Berufe 1992 entnehmen, die derzeit auch für den Mikrozensus verwendet wird. ${ }^{7}$ Auf dieser Skala, die von 30 Punkten für mithelfende Familienangehörige in der Landwirtschaft und für Landarbeitskräfte bis

\footnotetext{
${ }^{7}$ Davies u. Guppy (1997) versuchen den beruflichen Nutzen und Status anhand der durchschnittlichen Gehälter der über das Studium erreichbaren Berufe festzumachen. Dieses Vorgehen hat zunächst den Nachteil, dass über das aktuelle Einkommen die Erwerbschancen und die monetären Humankapitalrenditen grob indiziert werden, aber nicht der soziale Status, der sich aus der Klassenlage oder Schichtzugehörigkeit ergibt. Des Weiteren ist das Durchschnittsgehalt im Vergleich zum Lebenseinkommen ein unzureichender Indikator, da Ergebnisse für Erwerbseinkommen vom Messzeitpunkt in der berufliche Karriere abhängen (vgl. Becker u. Schömann 1996). Ein Anwalt in der Rechtsabteilung einer großen Firma verdient mit geringerer Berufserfahrung weniger als sein Kollege mit einer längeren Betriebszugehörigkeit. Ebenso ergeben sich aus einem Studienfach unterschiedliche Berufe oder berufliche Tätigkeiten, die in gleicher oder unterschiedlicher Weise vergütet werden. Beispielsweise erhalten Hochschullehrer - unabhängig vom vertretenen Fach - bei gleicher Berufserfahrung, bei gleichem Dienstalter und im gleichen Bundesland dasselbe Grundgehalt.
}

hin zu 216 Punkten für Zahnärzte reicht, wird Ärzten (191,3 Punkte; Tierärzte: 138,9 Punkte), Apothekern $(207,2)$ und Juristen (Richter, Staats- oder Amtsanwälte: 138,2 Punkte; Rechtsvertreter oder Rechtsberater 145,7 Punkte) das höchste Prestige beigemessen. Nicht selten sind Juristen in der Politik als Abgeordnete, Minister oder Wahlbeamte mit einem Prestigewert von 153,5 Punkten tätig. Des Weiteren haben Hochschullehrer und verwandte Berufe mit 152,5 Punkten ebenfalls ein hohes Prestige, sodass Akademiker wegen dem Statuserhalt ein hohes Interesse haben, dass ihre Kinder ebenfalls die akademische Karriere einschlagen. ${ }^{8}$

Aufgrund der unterschiedlichen, curricular (Studienpläne) und organisatorisch vorgegebenen (Studienordnungen und Prüfungsorganisation) Regelstudienzeiten variieren über die Studiendauer und die Studienaufwendungen zusätzlich zum Aufwand und zu den Kosten, die Kinder bis zum Abitur $\mathrm{zu}$ bringen und studieren $\mathrm{zu}$ lassen die direkten Ausbildungskosten zwischen den einzelnen Studienfächern. Hierbei sind die unteren Sozialschichten im Nachteil. Weil ein Medizinstudium in der Regel rund zwölf Semester andauert, sind damit höhere finanzielle Aufwendungen verbunden als für andere Studienfächer. Zwar liegt für Jura die Regelstudienzeit bei zehn Semestern, aber die notwendigen Repetitorien sowie teuere Bücher sind zusätzliche Kostenfaktoren, welche Abiturienten aus den einkommensschwächeren Sozialschichten vom Jurastudium abschrecken könnten (Hansen 1997): Lang andauernde Studienzeiten (Medizin) und finanziell aufwändige Studienrichtungen (Jura) dürften wegen der zu erwartenden Kosten in der Regel Studienberechtigte aus unteren Sozialschichten abschrecken, sodass Studierende aus mittleren und höheren Sozialschichten in diesen Studienrichtungen überrepräsentiert sind.

Neben den unmittelbar erwarteten Kosten für das Studium von bestimmten Fächern sind langfristige Kosten zu berücksichtigen, die mit der soziokulturellen Distanz von Elternhäusern zu den Universitäten im Allgemeinen und einigen Studienfächern im Besonderen korrelieren

\footnotetext{
${ }^{8}$ Beispielsweise erhalten Naturwissenschaftler mit 139,8 Punkten eine hohe Wertschätzung, gefolgt von Psychologen, Wirtschafts- und Sozialwissenschaftlern sowie den Statistikern, Marktforschern und verwandten Berufen mit jeweils 135,7 Punkten. Den Geistes- und Erziehungswissenschaftlern oder Wissenschaftlern ohne nähere Angabe werden 122,2 Punkte zugeordnet. Ein vergleichbares oder geringfügig geringeres erhalten Physiker und Mathematiker (125,2 Punkte), Chemiker bzw. Chemieoder Verfahrensingenieure (123,9 Punkte), Theologen bzw. Geistliche (122 Punkte) und Architekten (111,7 Punkte). Die Prestigewerte streuen für die Ingenieure von 88,3 Punkten (Wirtschafts- bzw. REFA-Ingenieure) bis 111,7 Punkte für Bauingenieure. Diejenigen für Informatiker und andere DV-Berufe liegen bei 79,6 Punkten. Wegen der Überschneidung in der MP-Skala ist eine Hierarchisierung von Studienfächern nicht sinnvoll. Sinnvoller scheint die Interpretation der Studienfächer als ,gate keeper“ für die Klassenlage bzw. Schichtzugehörigkeit als Indikator für Sozialstatus oder Statuserhaltungsmotiv.
} 
(Becker 2009; Böttcher et al. 1988, S. 13f.; Boudon 1974). Je weiter das Bildungsniveau des Elternhauses von den Hochschulen mit den angebotenen Studienfächern entfernt ist, desto größer sind auch die zu erwartenden Kosten (direkte und indirekte Ausbildungskosten, Transaktionskosten, Opportunitätskosten etc.), die Kinder über alle Hürden in der Schulbildung an die Universitäten oder Fachhochschulen zu bringen. Umgekehrt gilt natürlich, dass Angehörige der oberen Dienstklasse (insb. Akademiker und Professionelle) mit einer geringen Distanz zum System tertiärer Bildung aufgrund ihrer Vertrautheit mit Hochschulen und ihrem Studienfachkanon geringe Informations- und Transaktionskosten haben. ${ }^{9}$ Ebenso erwarten sie wegen ihrer privilegierten finanziellen Ausstattung vergleichsweise geringe Bürden durch anfallende Ausbildungskosten.

Diese Kostenerwartungen differieren deutlich zwischen den Sozialschichten von gering für die obere Dienstklasse bis sehr hoch für die Arbeiterklassen. Ähnliches gilt für die Studienfächer. Je größer die kulturelle Distanz des Elternhauses - gemessen am schulischen und beruflichen Bildungsniveau der Eltern - zu einem bestimmten Studienfach, desto höher erscheinen die Aufwendungen für dieses Studienfach. Diese Kosten können in Abhängigkeit der sozialen Herkunft minimiert werden, wenn Studienfächer mit einer möglichst geringen soziokulturellen Distanz gewählt werden. Diese Minimierung erfolgt für Bildungsaufsteiger dadurch, dass sie Studienfächer wählen, die eine inhaltliche Nähe zu den Berufen des Elternhauses aufweisen: Für Kinder von Medizinern oder Juristen ist daher das Studium von Medizin bzw. Jura interessant, während für Kinder von Landwirten das Studium der Agrarwissenschaft und Forstwirtschaft nahe liegt. Kinder von Prokuristen oder Unternehmern müssten sich daher eher für ein wirtschaftswissenschaftliches Studium interessieren (Böttcher et al. 1988, S. 12). Zudem sind für Kinder von mittelständischen oder größeren Unternehmern die Disziplinen interessant, die eine Nähe zum Produkt des elterlichen Unternehmens aufweisen (Beispiel: Maschinenbau für Maschinenhersteller oder Chemie für Chemikalienproduzenten).

\subsection{Zwischenfazit}

Insgesamt wird aus dem dargestellten Zusammenspiel primärer und sekundärer Herkunftseffekte deutlich, warum sich das Handlungsset von Oberschichten auf ein Minimum von Studienfächern beschränkt, und worauf ihr ,Anlagesinn für die ,richtigen“ Ausbildungsgänge“ beruht. Des Weiteren

\footnotetext{
${ }^{9}$ Die geringe soziokulturelle Distanz zum System tertiärer Bildung und zu bestimmten Studienfächern kann unter Umständen zur Verbesserung von Erfolgswahrscheinlichkeiten führen. Beispielsweise sind akademisch gebildete Eltern eher in der Lage, ihren Kindern im Studium entsprechende Unterstützung und Tipps zu geben, um deren Studienerfolg zu garantieren.
}

ist theoretisch begründet nachvollziehbar, warum die Studienfachwahl ein Spezialfall der intergenerationalen Berufsund Statusvererbung ist. Ebenso wird verständlich, worin die Affinität für einen fachspezifischen Lebensstil und der Studienfachkultur (Georg 2005, S. 62) besteht, warum der individuelle herkunftsbedingte „Habitus“ und kognitive Stil (Georg 2005, S. 62) sowie das herkunftsspezifische Streben nach mehr oder weniger Distinktion (Bourdieu 1984) mit der Studienfachwahl korreliert, und wie internalisierte Werte und die institutionalisierte Fachkultur (Windolf 1990) zusammenkommen.

So wird auch einleuchtend, welche Rolle individuelle, zwischen den Sozialschichten differierende Neigungen, Motivation (wie etwa Interesse an Wissenserwerb oder Selbstverwirklichung, Bildung an sich) und - daraus abgeleitet - das fachwissenschaftliche Interesse als notwendige, aber nicht hinreichende Gründe für eine Ausbildungsund Berufswahl spielen. Aus der Sicht von Bourdieu u. Passeron (1978) könnte argumentiert werden, dass die Belegung von Leistungskursen in der gymnasialen Oberstufe entweder Ausdruck individueller Neigungen (,,kultureller Habitus") ist oder dass diese die Abiturienten in der Art und Weise sozialisieren, dass sie die Studienfachwahl hochgradig determinieren. Abgesehen davon, dass nicht erklärt wird, warum dann nicht die meisten Abiturienten studieren, wenn sie durch einen Leistungskurs kulturell geprägt werden und dabei das Studienfach wählen, das am ehesten den Leistungskursen entspricht, also warum sich einige Abiturienten für andere Alternativen entscheiden, ist dem strukturell-individualistischen Erklärungsansatz nach Boudon (1974) zufolge eine alternative Argumentation plausibel. Die Auswahl der Leistungskurse in der gymnasialen Oberstufe ist eine frühe Investition in das avisierte Studienfach oder in eine bestimmte nichttertiäre Berufsausbildung und daher Bestandteil des Statuserhaltungsmotivs. Es wird sicherlich nicht von der Hand zu weisen sein, dass belegte Leistungskurse - sofern noch keine Studienfachentscheidung gefallen ist oder nur vage inhaltliche Vorstellungen für eine bestimmte Ausbildung bestehen zur definitiven Ausbildungsentscheidung beitragen. Für die Kinder von Medizinern oder Juristen dürfte die Auswahl der Leistungskurse strategisch mit dem Ziel erfolgen, frühzeitig in ein Medizin- oder Jurastudium zu investieren. Somit wird die Auswahl von Leistungskursen als ein hinreichender Zwischenschritt für die Ausbildungsentscheidung und ihre inhaltliche Ausrichtung angesehen, der allenfalls bedingt zur Erklärung des Zusammenhangs von sozialer Herkunft und Studienfachwahl beiträgt.

\subsection{Hypothesenbildung}

Aus dieser handlungs- und werterwartungstheoretischen Argumentation heraus lässt sich folgende Hypothese ableiten: 
Bei Kontrolle primärer und sekundärer Herkunftseffekte werden die Bruttoeffekte der sozialen Herkunft für die Entscheidung eines bestimmten Studienfachs insignifikant. Wenn diese Herkunftseffekte unbedeutsam werden, dann sind sie nicht nur statistisch ,erklärt“, sondern damit hat sich der herangezogene Erklärungsansatz empirisch bewährt. Inhaltliche Neigungen und Interessen an sich werden kaum zusätzlich zur empirischen Auflösung der vorliegenden Fragestellung beitragen. Denn diese ergeben sich zum einen aus der Sozialisation und zum anderen aus dem Motiv des Statuserhalts selbst.

\section{Daten, Variablen und statistisches Verfahren}

\subsection{Datenbasis}

Die empirischen Analysen basieren auf Daten von Abiturientenbefragungen, die in Sachsen seit 1996 alle zwei Jahre im Auftrag des Sächsischen Staatsministeriums für Kultus in sächsischen Regionalschulbezirken (Bautzen, Chemnitz, Dresden, Leipzig und Zwickau) durchgeführt werden. Ziel der Befragung ist es, die Entscheidungsfindung der sächsischen Abiturientinnen und Abiturienten zu ihren nachschulischen Bildungs- und Berufswegen ca. drei Monate vor dem Abitur zu untersuchen (Wolter et al. 2006, S. 3). Die Grundgesamtheit umfasst alle Schülerinnen und Schüler in der 12. Klasse der allgemeinbildenden Gymnasien, der 13. Klasse der beruflichen Gymnasien und der 12. Klasse der Fachoberschulen. Für die Stichproben wurden jeweils $10 \%$ der Schüler und Schülerinnen in den Abschlussklassen an zufällig ausgewählten Schulen herangezogen. In den Umfragen wurden diese Schüler und Schülerinnen schriftlich mit standardisierten Fragebögen befragt. Aufgrund der für unsere Fragestellung relevanten Informationen können wir lediglich auf die seit 2000 durchgeführten Studien zurückgreifen. Die Größe der Grundgesamtheiten, der Stichproben und der Rücklaufquoten sind in Tabelle 1 dokumentiert.

Aus diesen vier Jahrgängen werden im Folgenden nur Befragte berücksichtigt, die sich bereits entschieden haben, was sie unmittelbar nach dem Abitur und gegebenenfalls nach Ableistung des Zivil- oder Militärdienstes tun werden.

Tabelle 1 Grundgesamtheit, Stichproben und Rücklaufquoten der bisherigen Erhebungen

\begin{tabular}{lcccc}
\hline & 2000 & 2002 & 2004 & 2006 \\
\hline Grundgesamtheit & 20.464 & 21.003 & 19.926 & 19.559 \\
Stichproben & 1937 & 2028 & 1883 & 2053 \\
Rücklaufquoten & $78,4 \%$ & $80,1 \%$ & $78,1 \%$ & $78,8 \%$ \\
\hline
\end{tabular}

Quelle: Wolter et al. (2006, S. 4)
Unberücksichtigt bleiben unentschlossene Befragte. Eigene Analysen haben ergeben, dass sich die Ergebnisse nicht signifikant verändern, wenn Unentschlossene eingeschlossen werden (zu Details: Becker u. Hecken 2009a,b). Zudem belegen die HIS-Studienberechtigten-Studien (Heine u. Willich 2006) eine hohe Übereinstimmung der erfragten Ausbildungs- und Berufsentscheidung mit dem tatsächlich realisierten Übergang, sodass die erfassten Ausbildungsentscheidungen hochgradig mit dem späteren Handeln der Befragten korrespondieren. Des Weiteren belegen Heine et al. (2007, S. 60), dass sich die Mehrheit der Studienberechtigten auch für das beabsichtigte Studienfach immatrikulieren.

\subsection{Definition der abhängigen Variablen}

Die abhängige Variable ist die individuelle Entscheidung, im Anschluss an das Abitur entweder an einer Hochschule (Universität oder Fachhochschule) ein bestimmtes Studienfach zu studieren oder eine nichttertiäre Ausbildung zu beginnen. Theoriegeleitet wird bei den Studienfächern stellvertretend für prestigeträchtige Disziplinen, professionelle Berufe und daher für Abiturienten aus den höheren Sozialschichten interessante Studienrichtungen - zunächst zwischen Medizin (Human- und Veterinärmedizin sowie Pharmazie) und Jura unterschieden (Heine u. Scheller 2005, S. 33; Multrus 2006). ${ }^{10}$ Des Weiteren werden Studienfächer wie Elektrotechnik, Maschinenbau und Verfahrenstechnik oder Erziehungs- und Sozialwissenschaften (inklusive Psychologie) einbezogen, die besonders für soziale Bildungsaufsteiger aus den Arbeiterklassen interessant zu sein scheinen (s. Heine u. Scheller 2005, S. 34). Und schließlich werden Studienrichtungen wie Wirtschaftswissenschaften und Naturwissenschaften (Biologie, Chemie, Physik und Geowissenschaften) berücksichtigt, denen nachgesagt wird, dass sie nicht eindeutig als Studienfächer für bestimmte soziale Schichten identifiziert werden können (s. Reimer u. Pollak 2005). Nach Heine u. Scheller (2005, S. 35) sind sie wie die Geisteswissenschaften (Kultur- und Sprachwissenschaften, Geschichte, Kunst und Gestaltungswissenschaft), Architektur und Bauwesen sowie Mathematik und Informatik eher eine „Domäne“ der Mittelschichten oder der oberen Dienstklasse. Disziplinen wie Agrar-, Forst- und Ernährungswissenschaft, Verkehrswissenschaft, Musik, Sportwissenschaft und nicht spezifizierte Studienfächer werden zu einer weiteren Kategorie zusammengefasst. Weitere Differenzierungen nach Studiengängen, um etwa

\footnotetext{
${ }^{10}$ Pharmazie - ein naturwissenschaftliches Studienfach mit einer gewissen Nähe zu Biologie und Chemie - wird zu Medizin gerechnet, weil in der Erhebung im Jahre 2000 diese Kategorisierung vorgenommen wurde. In den nachfolgenden Erhebungen wurde separat nach Medizin und Pharmazie als Studienrichtung gefragt. Aufgrund geringer Fallzahlen für diese beiden Studienfächer wird die Definition aus dem Jahre 2000 für die darauffolgenden Beobachtungszeitpunkte beibehalten.
} 
Tabelle 2 Studienfachwahl und Zeitpunkt der Ausbildungs- und Studienentscheidung in Abhängigkeit von sozialer Herkunft (odds ratios, geschätzt mit binärer bzw. multinomialer LogitRegression)

\begin{tabular}{lcccc}
\hline & $\begin{array}{c}\text { Noch kein } \\
\text { Studienfach } \\
\text { ausgewählt }\end{array}$ & $\begin{array}{c}\text { Vor Eintritt in } \\
\text { gymnasiale Oberstufe } \\
\text { bzw. Berufsgymnasium }\end{array}$ & $\begin{array}{c}\text { Schon im letzten } \\
\text { Schuljahr }\end{array}$ & $\begin{array}{c}\text { In der ersten Hälfte } \\
\text { des Schuljahres }\end{array}$ \\
\hline Arbeiterklassen & 1 & 1 & 1 & 1 \\
Mittelschichten & 0,96 & 0,89 & 1,07 & 1,09 \\
Obere Dienstklasse & $1,57^{*}$ & 1,18 & 1,08 & \\
Pseudo-R & 0,004 & & 0,001 & \\
Fallzahl & 3728 & & $30,6 \%-33,2 \%-26,7 \%-(9,5 \%)$ \\
Quote & $5,7 \%$ & 3 & \\
\hline
\end{tabular}

* Mindestens $p \leq 0,05$

Quelle: Abiturientenbefragung in Sachsen 2002-2006 - eigene Berechnungen
Lehramtsstudierende identifizieren zu können, sind mit den verwendeten Daten nicht möglich.

Wie zuvor gesehen, ist die Hierarchisierung von Studienfächern mittels durchschnittlichem Einkommen (Davies u. Guppy 1997) oder Prestige als Bildungsrendite problematisch. Beispielsweise differieren die Gehälter von Ärzten beträchtlich: So verdienen in der Regel Tierärzte durchschnittlich weniger als Allgemeinärzte, Landärzte weniger als Zahnärzte oder Gynäkologen weniger als Ärzte in der Mikrochirurgie, in der plastischen oder der ästhetischen Chirurgie. Gleiches gilt auch für Juristen. Auch die Verwendung von Prestigeskalen wie etwa die MPS von Wegener (1988) ist für unseren Fall wenig geeignet, da der Prestigewert zwar der beruflichen Tätigkeit, aber nicht einem Studienfach eindeutig zugeordnet werden kann. Zudem kennen wir die genaue Bezeichnung der nichttertiären Berufsausbildung (etwa den ISCO) nicht, sodass dieser kein Prestigewert zugeordnet werden kann. Da mit einem bestimmten Studienfach mehrere berufliche Tätigkeiten möglich sind, kann - da wir es mit Abiturienten zu tun haben und daher noch keine Daten über ihre spätere berufliche Tätigkeit verfügen - ebenfalls keine eindeutige Zuordnung von Prestigewerten vorgenommen werden. Am sinnvollsten wären Informationen zum Zusammenhang von Studienfach und Übergang in eine Klassenposition. Da diese nicht vorliegen, belassen wir es bei der zuvor gewählten Kategorisierung der Studienfächer und konzentrieren uns bei der Interpretation der Daten auf die Studienfächer wie Medizin und Jura.

Die Referenzkategorie der abhängigen Variablen ist die Entscheidung für eine nichtakademische Berufsausbildung an der Berufsakademie bzw. für eine nichttertiäre berufliche Lehre. ${ }^{11}$ In Bezug auf unsere Fragestellung ist eine große

\footnotetext{
${ }^{11}$ Weil der Anteil derjenigen, die sofort erwerbstätig werden wollen, weniger als $4 \%$ beträgt, werden diese Personen auch aus statistischen Gründen geringer Fallzahlen nicht weiter berücksichtigt. Kürzlich erschienene Studien dokumentieren für Deutschland eine Quote von 5-6\% von Abiturienten, die ein halbes Jahr nach Erwerb der Hochschulreife ohne weitere Ausbildung erwerbstätig wurden (Heine et al. 2008).
}

(mehr als ein Drittel der Befragten) wie heterogene Referenzkategorie für den nichttertiären Ausbildungsbereich (vgl. Becker u. Hecken 2007) sicherlich nicht unproblematisch. Eine mögliche Problemlösung wäre die theoretische Vorannahme, dass die Studienfachwahl synchron mit der Studienentscheidung anstatt sequenziell (zuerst Studium, dann Studienfach) einhergeht (vgl. Becker 2000b). Ob diese Vorannahme empirisch zutreffend ist, haben wir mit für diese Problematik wenig geeigneten Querschnittsdaten der Abiturientenbefragung $\mathrm{zu}$ belegen versucht (Tabelle 2). Aus erhebungs- und datentechnischen Gründen kann nur der Befragungszeitraum zwischen 2002 und 2006 berücksichtigt werden. Zunächst wird der Frage nachgegangen, ob sich die studierwilligen Befragten bis zum jeweiligen Befragungszeitpunkt im März und April - also drei Monate vor dem Abitur - für ein Studienfach entschieden haben. Rund 93\% dieser Befragten wissen bereits, was sie studieren werden. Die soziale Herkunft hat keinen signifikanten Einfluss auf die Entschlossenheit einer Studienfachwahl. Dass mit rund 6\% ein kleiner Anteil dieser Befragten noch keine Studienfachwahl getroffen hat, könnte zunächst ein erster Hinweis für synchrone Ausbildungsentscheidungen sein. Diese Vermutung wird noch dadurch gestützt, dass rund ein Drittel der Befragten bereits vor dem Eintritt in die gymnasiale Oberstufe, ein weiteres Drittel bereits kurz nach dem Übergang in die gymnasiale Oberstufe und schließlich ein Drittel erst im aktuellen Schuljahr eine Studienentscheidung getroffen hatte. Entscheidend für unsere Fragestellung ist, dass lediglich ein äußerst geringer Prozentsatz der Befragten erst nach dem Befragungszeitpunkt eine Studienfachwahl treffen wird. Dies trifft dann vor allem für Abiturienten aus der oberen Dienstklasse zu, für die ein Hochschulstudium unabhängig vom Studienfach notwendig zu sein scheint, um den Status zu erhalten. Um diese Problematik der Abfolge von Studienentscheidung und Studienfachwahl abschließend zu klären, wären zeitlichkontinuierliche Längsschnittdaten notwendig. Solche Daten liegen unseres Wissens für Deutschland nicht vor. Angesichts der vorliegenden Daten gehen wir im Folgenden von einer synchronen Entscheidung der Abiturienten aus. 
Abgesehen davon, dass mit Querschnittsdaten nicht erschöpfend überprüft werden kann, ob die Studienentscheidung und Studienfachwahl bzw. die Entscheidung für eine nichttertiäre Berufsausbildung mit einem bestimmten Berufswunsch vornehmlich synchron erfolgt, erkauft man sich allerdings das statistisch-methodische Problem einer sozial selektiven Stichprobe, wenn nur die Studierwilligen oder Studienanfänger berücksichtigt werden. Diesen ,sample selection bias" könnte man mit dem zweistufigen, von Heckman (1979) vorgeschlagenen Verfahren korrigieren, indem man in einem ersten Schritt die Wahrscheinlichkeit schätzen würde, an der Universität oder Fachhochschule zu studieren. Im zweiten Schritt würde dann diese als unabhängige Variable (,propensity scores“) im Modell für die Studienfachwahl fungieren (vgl. Becker 2003). Weil für diese beiden Entscheidungen jeweils identische Mechanismen wirken und daher auch identische Modelle geschätzt werden müssten, träte für die Modellierung des zweiten Schrittes (Studienfachwahl) das Problem der Multikollinearität auf. Aufgrund dieser technischen Schwierigkeiten und auch wegen der hier verfolgten Fragestellung wird die Beibehaltung einer großen wie heterogenen Referenzkategorie (Abiturienten mit Entscheidung für nichttertiäre Ausbildung) als das geringste Übel angesehen.

\subsection{Operationalisierung der unabhängigen Variablen}

Die soziale Herkunft stellt auch für die Studienfachwahl eine zentrale erklärende Variable dar. In den sächsischen Abiturientenbefragungen werden weder die genaue Bezeichnung des erlernten Berufs oder der beruflichen Tätigkeit der Elternteile noch die Studienfächer von Eltern mit einem Hochschulabschluss festgehalten. Deswegen kann unter Heranziehung der beruflichen Stellung der Eltern der Statuserhalt über Studienfächer und Berufsvererbung nur in einem eingeschränkten Maße untersucht werden. Um die Modellierungen übersichtlich zu halten, wird die berufliche Stellung nach dem Kategorienschema von Becker u. Hecken (2008) in drei soziale Klassen zusammengefasst: Die Arbeiterklassen umfassen un- und angelernte Arbeiter, Facharbeiter, Vorarbeiter und Meister. Die Mittelschichten schließen Angestellte mit einfacher Tätigkeit (z.B. Verkaufsberufe), Beamte im einfachen und mittleren Dienst, Angestellte mit qualifizierter (z. B. Sachbearbeiter) oder hochqualifizierter Tätigkeit, Beamte im gehobenen Dienst sowie Selbstständige ein. Die obere Dienstklasse schließlich besteht aus Angestellten mit einer Leitungsfunktion (z. B. Prokurist), Angestellten mit Führungsaufgaben (z. B. Geschäftsführer), Beamten im höheren Dienst (bzw. Richter) und akademischen bzw. freien Berufen (z. B. Arzt mit eigener Praxis, Rechtsanwalt). Es wird jeweils die höhere Kategorie der beiden Elternteile berücksichtigt. Ein fehlender Wert für ein Elternteil wird - sofern möglich - durch die Angabe des anderen Elternteils substituiert.

In Anlehnung an Opp u. Friedrichs (2002) beabsichtigen wir eine direkte Anwendung eines Rational-Choice-Ansatzes (vgl. Brüderl 2004). Daher versuchen wir, individuelle Präferenzen, Erwartungen und Evaluationen direkt zu messen (Manski 2004). Bei Sekundäranalysen fehlen einerseits entsprechende Messungen (Friedrichs et al. 1993): In unserem Fall verfügen wir weder über Informationen zu den übergeordneten Zielen wie materielles Wohlergehen, soziale Anerkennung oder Statuserhalt noch über optimale Operationalisierungen erklärender Variablen (Tabelle 3). Abgesehen von theoretisch plausiblen und empirisch fundierten Brückenhypothesen bürgen neben extensiven Überprüfungen der Proxy-Variablen - insbesondere derjenigen, welche das Statuserhaltungsmotiv abbilden sollen - erfolgreiche Anwendungen für eine ausreichende Validität der herangezogenen Indikatoren (Becker u. Hecken 2007, 2008, 2009a,b; Maaz 2006; Becker 2000a). So korrelieren diese Indikatoren, wie theoretisch erwartet und in Tabelle 4 dokumentiert, signifikant mit der sozialen Herkunft (vgl. Becker u. Hecken 2009a,b). Die indirekte Operationalisierung des Entscheidungsprozesses schränkt die Aussagekraft unserer Ergebnisse in gewisser Weise ein, sodass angesichts dieser methodischen Problematik eine zurückhaltende Interpretation der Befunde angebracht ist. Auch ist es mit den herangezogenen Daten wegen fehlender Informationen nicht möglich, das Modell der Frame-Selektion (Kroneberg 2005) empirisch anzuwenden, um weitergehende Befunde zum Zusammenhang von sozialer Herkunft und Studienfachwahl vorzulegen.

In Tabelle 4 sind die Verteilungen der erklärenden Variablen nach sozialer Herkunft dargestellt. Wie theoretisch erwartet, liegen deutliche primäre Herkunftseffekte vor. So stufen sich Abiturienten aus den Arbeiterklassen eher als schlechte Schüler ein als diejenigen aus den mittleren und höheren Sozialschichten. Sie haben auch eine geringere Erfolgserwartung und sind weniger überzeugt davon, ein Studium erfolgreich bewältigen zu können. Diese Unterschiede nach sozialer Herkunft sind jeweils signifikant.

Ebenso sind die anhand des höchsten Bildungsabschlusses der Eltern gemessenen sozialen Distanzen zum System der tertiären Bildung für die drei Sozialschichten signifikant verschieden: Je höher die Platzierung des Elternhauses in der sozialen Schichtung, desto eher haben die Eltern akademische Abschlüsse, und desto geringer sind die sozialen Distanzen zu den Hochschulen.

Wie von Esser (1999) theoretisch erwartet und bereits mehrfach empirisch belegt (Becker u. Hecken 2009a), unterscheiden sich bei der subjektiven Einschätzung des beruflichen Bildungsertrags $U$ die einzelnen sozialen Schichten nicht substanziell voneinander. Erst auf dem 10\%-Niveau lassen sich Schichtunterschiede festmachen. Hingegen 
Tabelle 3 Operationalisierung der abhängigen und unabhängigen Variablen

\begin{tabular}{ll}
\hline Theoretisches Konstrukt & Messung im Original \\
\hline Ausbildungsentscheidung & $\begin{array}{l}\text { Frage: „Was werden Sie wahrscheinlich } \\
\text { nach dem Erwerb des Abiturs bzw. der } \\
\text { Fachhochschulreife (von Wehrpflicht, } \\
\text { Zivildienst u. ä. abgesehen) tun?“ }\end{array}$
\end{tabular}

Subjektiv erwarteter beruflicher Nutzen $U$

Subjektiv erwarteter Betrag des Statusverlusts $-S V$

Subjektiv erwartete Wahrscheinlichkeit für Statusverlust $c$

Subjektiv erwartete Kosten C für ein Studium

Subjektiv erwarteter Studienerfolg $p$

Selbstbewertete Schulleistung $P$

Soziale Distanz $S D$

Soziale Herkunft
Frage: ,Wie schätzen Sie die Berufsaussichten für Akademiker allgemein ein?“

Frage: ,Wie schätzen Sie ganz allgemein die Berufsaussichten für Absolventen beruflicher Ausbildungswege ohne Studium ein?“"

Frage: „Glauben Sie, dass Sie bei einer Berufsausbildung in Verbindung mit beruflicher Weiterbildung die gleichen beruflichen Chancen haben wie mit einem Studienabschluss?“"

Frage: „Haben die Kosten des Studiums Einfluss auf Ihre Entscheidung für oder gegen ein Studium?“

Frage: „Fühlen Sie sich aufgrund Ihrer schulischen Vorbildung in der Lage, ein Studium erfolgreich durchzuführen?“"

Frage: ,Wenn Sie Ihren schulischen Leistungsstand einschätzen, wie würden Sie sich einordnen?"

Frage: ,Welchen (höchsten) beruflichen Abschluss haben Ihre Eltern?"
Kodierung

$1=$ nichtakademische Berufsausbildung (Berufsakademie oder berufliche Lehre) $-2=$ Anderes Studienfach an Fachhochschule oder Universität (Agrar-, Forst- und Ernährungswissenschaft, Verkehrswissenschaft, Musik, Sportwissenschaft und sonstige Fächer) $-3=$ Medizin (Human- und Veterinärmedizin sowie Pharmazie) $-4=$ Jura $-5=$ Elektrotechnik und Maschinenbau bzw. Verfahrenstechnik $-6=$ Sozialwissenschaften $7=$ Wirtschaftswissenschaften $-8=$ Naturwissenschaften $9=$ Geisteswissenschaften $-10=$ Architektur und Bauwesen $11=$ Mathematik und Informatik

$1=$ sehr schlecht $-5=$ sehr gut

$1=$ sehr niedrig $-5=$ sehr hoch

(ursprüngliche Kodierung von , $1=$ sehr hoch $-5=$ sehr niedrig“ umgepolt)

$1=$ sehr niedrig $-5=$ sehr hoch

(ursprüngliche Kodierung von , $1=$ sehr hoch $-5=$ sehr niedrig“ umgepolt)

$1=$ sehr niedrig $-5=$ sehr hoch

$1=$ hoch $-0=$ niedrig bzw. weiß nicht

$1=$ sehr schlecht $-4=$ sehr gut

Abstand zwischen dem höchsten beruflichen Bildungsabschluss eines Elternteils und der Universität: $5=$ keinen beruflichen Abschluss, 4 = Lehre bzw. Facharbeiterabschluss, $3=$ Meisterprüfung, 2 = Abschluss einer Fach- bzw. Ingenieurschule und schließlich $1=$ Universitäts- bzw. Fachhochschulabschluss

$1=$ nicht vorhanden $-5=$ sehr hoch

Arbeiterklasse, Mittelschichten und obere Dienstklasse (vgl. Becker u. Hecken 2008) ist der zuletzt ausgeübte Beruf Ihrer Eltern zuzuordnen?"“

unterscheiden sich die Arbeiterkinder deutlich von den Abiturienten aus den mittleren und besonders von Kindern aus höheren Sozialschichten im Statuserhaltungsmotiv - sprich: in der subjektiven Erwartung von Wahrscheinlichkeiten und Beträgen von Statusverlusten infolge eines Verzichts auf ein Hochschulstudium. Gemäß den erwartungstheoretischen Ansätzen sind die Abiturienten aus den mittleren und höheren Sozialschichten eher davon überzeugt, dass bei suboptimalen Bildungsentscheidungen der intergenerationale Statuserhalt nicht mehr garantiert ist. Diese subjektiv erwarteten Wahrscheinlichkeiten eines Statusverlusts in- folge eines Verzichts auf ein Hochschulstudium trennt die Sozialschichten im Unterschied zum subjektiv erwarteten Betrags eines Statusverlustes infolge einer suboptimalen Ausbildungsentscheidung statistisch signifikant voneinander. In der Logik der sozialen Ungleichheit erfolgt auch die subjektive Einschätzung der Kosten für ein Studium: Arbeiterkinder schätzen die Kosten signifikant höher ein als Absolventen aus den Mittel- und Oberschichten.

Insgesamt entsprechen diese Befunde den theoretischen Aussagen von Boudon (1974) zu primären und sekundären Herkunftseffekten (vgl. Definition der Indikatoren für 
Tabelle 4 Verteilung von Determinanten der Ausbildungsentscheidung und Studienfachwahl nach sozialer Herkunft (Mittelwert und in Klammern: Standardabweichung bzw. Standardfehler)

\begin{tabular}{|c|c|c|c|c|}
\hline & Arbeiterschichten & Mittelschichten & Obere Dienstklasse & Insgesamt \\
\hline $\begin{array}{l}\text { Indikatoren für primäre Herkunftseffekte } \\
\text { Schulleistung } P \\
(1=\text { sehr schlecht }-4=\text { sehr gut })\end{array}$ & $\begin{array}{c}2,58 \\
(0,65 / 0,02)\end{array}$ & $\begin{array}{c}2,64 \\
(0,68 / 0,01)\end{array}$ & $\begin{array}{c}2,68 \\
(0,68 / 0,03)\end{array}$ & $\begin{array}{c}2,63 \\
(0,67 / 0,01)\end{array}$ \\
\hline $\begin{array}{l}\text { Erwarteter Studienerfolg } p \\
(0=\text { niedrig }-1=\text { hoch })\end{array}$ & $\begin{array}{c}0,55 \\
(0,50 / 0,01)\end{array}$ & $\begin{array}{c}0,61 \\
(0,49 / 0,01)\end{array}$ & $\begin{array}{c}0,65 \\
(0,48 / 0,02)\end{array}$ & $\begin{array}{c}0,60 \\
(0,49 / 0,01)\end{array}$ \\
\hline $\begin{array}{l}\text { Indikatoren für sekundäre Herkunftseffekte } \\
\text { Beruflicher Nutzen } U \\
(1=\text { sehr niedrig }-5=\text { sehr hoch })\end{array}$ & $\begin{array}{c}3,43 \\
(1,17 / 0,03)\end{array}$ & $\begin{array}{c}3,41 \\
(1,14 / 0,02)\end{array}$ & $\begin{array}{c}3,51 \\
(1,09 / 0,04)\end{array}$ & $\begin{array}{c}3,41 \\
(1,14 / 0,02)\end{array}$ \\
\hline $\begin{array}{l}\text { Erwartung eines Statusverlusts } c \\
(1=\text { sehr niedrig }-5=\text { sehr hoch })\end{array}$ & $\begin{array}{c}3,20 \\
(1,05 / 0,03)\end{array}$ & $\begin{array}{c}3,28 \\
(1,03 / 0,02)\end{array}$ & $\begin{array}{c}3,37 \\
(1,03 / 0,04)\end{array}$ & $\begin{array}{c}3,27 \\
(1,04 / 0,01)\end{array}$ \\
\hline $\begin{array}{l}\text { Erwarteter Betrag des Statusverlusts }-S V \\
(1=\text { sehr niedrig }-5=\text { sehr hoch })\end{array}$ & $\begin{array}{c}2,21 \\
(0,97 / 0,03)\end{array}$ & $\begin{array}{c}2,23 \\
(0,96 / 0,02)\end{array}$ & $\begin{array}{c}2,26 \\
(0,95 / 0,04)\end{array}$ & $\begin{array}{c}2,23 \\
(0,96 / 0,01)\end{array}$ \\
\hline $\begin{array}{l}\text { Erwartete Kosten für Studium } C \\
(1=\text { sehr niedrig }-5=\text { sehr hoch })\end{array}$ & $\begin{array}{c}3,12 \\
(1,30 / 0,03)\end{array}$ & $\begin{array}{c}2,81 \\
(1,31 / 0,02)\end{array}$ & $\begin{array}{c}2,60 \\
(1,31 / 0,05)\end{array}$ & $\begin{array}{c}2,87 \\
(1,31 / 0,02)\end{array}$ \\
\hline $\begin{array}{l}\text { Soziale Distanz } S D \\
(1=\text { nicht vorhanden }-5=\text { sehr hoch })\end{array}$ & $\begin{array}{c}3,57 \\
(0,78 / 0,02)\end{array}$ & $\begin{array}{c}2,45 \\
(1,28 / 0,02)\end{array}$ & $\begin{array}{c}1,66 \\
(0,98 / 0,04)\end{array}$ & $\begin{array}{c}2,65 \\
(1,28 / 0,02)\end{array}$ \\
\hline
\end{tabular}

Quelle: Abiturientenbefragung in Sachsen 2000-2006 - eigene Berechnungen

die beiden Herkunftseffekte in Tabelle 4), dass die Arbeiterkinder vergleichsweise ungünstigere Schulleistungen und Erfolgswahrscheinlichkeiten aufweisen und Angehörige der Arbeiterklassen höhere Kosten infolge fortgesetzter Bildung ihrer Kinder befürchten und in geringerem Maße vom Nutzen höherer Bildung für den Statuserhalt überzeugt sind.

\subsection{Statistische Verfahren}

In Anlehnung an die Kritik von Lucas (2001) an der von Mare (1980) vorgeschlagenen statistischen Modellierung von Ausbildungsentscheidungen und Bildungsübergängen wird für die statistische Modellierung des Entscheidungsprozesses sowohl die binäre als auch die multinomiale logistische Regression angewandt (Long 1997). Die Verwendung dieser Modellierung kann sowohl verteilungstheoretisch als auch entscheidungstheoretisch begründet werden. So kann die Studienfachwahl mit einem handlungstheoretischen Ansatz der subjektiven Optimierung von Zielen rekonstruiert werden. Dieser probabilistische Erklärungsansatz kann für die empirische Anwendung mit der Logik und Statistik von logistischen Regressionen verknüpft werden (Urban 1993, S. 108-119).

Aus Gründen von Komplexitätsreduktion und Überschaubarkeit der Tabellen werden Schätzungen mittels logistischer Regressionen ausschließlich in Form von odds ratios dokumentiert. Sie geben jeweils das Chancenverhältnis für die miteinander verglichenen Gruppen wieder, einen bestimmten Zustand aufzuweisen oder ein Ereignis zu erfahren. Werte von 1 besagen, dass es keinen
Zusammenhang zwischen unabhängiger und abhängiger Variablen gibt. Bei Werten $>1$ liegen positive, und bei Werten $<1$ liegen negative Einflüsse erklärender Variablen auf die abhängige Variable vor.

\section{Empirische Befunde}

\section{1 Überprüfung des Grundmodells}

Von allen befragten Abiturienten, die bereits eine mehr oder weniger definitive Ausbildungs- bzw. Berufsentscheidung getroffen haben, haben sich fast zwei Drittel für ein Hochschulstudium (Universität oder Fachhochschule) entschieden (letzte Zeile für Modell 1 in Tabelle 5). Dieser Anteil setzt sich folgendermaßen nach Studienfächern zusammen: Rund 3\% dieser Abiturienten beabsichtigt, Medizin (Human- oder Tiermedizin bzw. Pharmazie) und rund $1 \%$ beabsichtigt, Jura zu studieren. Von den hier berücksichtigten Studienfächern finden die Geisteswissenschaften $(9,5 \%)$, die Sozialwissenschaften $(8,5 \%)$ und die Naturwissenschaften (8\%), gefolgt von Maschinenbau, Elektrotechnik und Verfahrenstechnik (6,5\%), Wirtschaftswissenschaft $(6,1 \%)$ und Mathematik bzw. Informatik (5,6\%) den häufigsten Zuspruch. Das Schlusslicht bilden Architektur und Bauwesen mit einem Anteil von rund 3\%. Fächer wie Agrar-, Forst- und Ernährungswissenschaft, Verkehrswissenschaft, Musik, Sportwissenschaft und weitere unspezifizierte Fächer weisen prozentuale Anteile von weniger als $3 \%$ auf und werden daher in der Kategorie 
Tabelle 5 Studienfachwahl nach sozialer Herkunft und Determinanten der Studienfachwahl ${ }^{1}$ - Multinomiale Logit-Regression (odds ratios)

\begin{tabular}{lllllllll}
\hline $\begin{array}{l}\text { Andere } \\
\text { Fächer }\end{array}$ & Medizin Jura & ET/MB & SoWi & WiWi & $\begin{array}{c}\text { Natur- } \\
\text { wissen- } \\
\text { schaften }\end{array}$ & $\begin{array}{c}\text { Geistes- } \\
\text { wissen- } \\
\text { schaften }\end{array}$ & $\begin{array}{c}\text { Architek- } \\
\text { tur/Bau- } \\
\text { wesen }\end{array}$ &
\end{tabular}

\begin{tabular}{|c|c|c|c|c|c|c|c|c|c|c|}
\hline \\
\hline \multicolumn{11}{|l|}{ Soziale Herkunft } \\
\hline Arbeiterklassen & 1 & 1 & 1 & 1 & 1 & 1 & 1 & 1 & 1 & 1 \\
\hline Mittelschichten & $1,35^{*}$ & 2,16 & 1,41 & 1,01 & $1,45^{*}$ & $1,54^{*}$ & $1,39^{*}$ & $1,40^{*}$ & 1,17 & 1,19 \\
\hline Obere Dienstklasse & $2,21^{*}$ & $4,95^{*}$ & $3,98^{*}$ & $1,67^{*}$ & $2,19^{*}$ & $2,09^{*}$ & $1,56^{*}$ & $1,84^{*}$ & 1,62 & 1,48 \\
\hline \% Anteile $(N=5.566)$ & $10,0 \%$ & $3,2 \%$ & $1,0 \%$ & $6,5 \%$ & $8,5 \%$ & $6,1 \%$ & $8,0 \%$ & $9,5 \%$ & $3,3 \%$ & $5,6 \%$ \\
\hline
\end{tabular}

Modell 2: Pseudo- $\mathrm{R}^{2}(\mathrm{McFadden})=0,089$

Soziale Herkunft

Arbeiterklassen

Mittelschichten

Obere Dienstklasse

Studienfachwahl

Schulleistung

Beruflicher Nutzen

$P$ (Statusverlust)

Betrag des Statusverlusts

Studienerfolg

Kosten für Studium

Soziale Distanz

$\begin{array}{cc}1 & 1 \\ 0,96 & 1,14 \\ 1,14 & 1,82\end{array}$

1
0,81
1,48

1
$0,66^{*}$
0,75

1
1,04
1,31

1
1,04
1,00

1
0,90
0,78

1
0,94
0,99

1
0,68

0,71

1

$0,67^{*}$

0,65

$\begin{array}{llllllllll}1,70^{*} & 4,48^{*} & 2,46^{*} & 1,33^{*} & 1,55^{*} & 2,27^{*} & 2,22^{*} & 1,66^{*} & 1,23 & 2,16^{*} \\ 1,25^{*} & 1,30^{*} & 1,56^{*} & 1,57^{*} & 1,19^{*} & 1,41^{*} & 1,39^{*} & 1,15^{*} & 1,10 & 1,50^{*} \\ 1,78^{*} & 1,86^{*} & 2,07^{*} & 2,26^{*} & 1,76^{*} & 2,12^{*} & 1,74^{*} & 1,63^{*} & 1,75^{*} & 1,87^{*} \\ 1,58^{*} & 1,48^{*} & 2,15^{*} & 1,80^{*} & 1,59^{*} & 1,41^{*} & 1,63^{*} & 1,59^{*} & 1,58^{*} & 1,54^{*} \\ 1,78^{*} & 1,48^{*} & 1,48 & 2,78^{*} & 2,04^{*} & 2,05^{*} & 2,01^{*} & 2,36^{*} & 2,49^{*} & 2,09^{*} \\ 0,80^{*} & 0,68^{*} & 0,60^{*} & 0,76^{*} & 0,84^{*} & 0,80^{*} & 0,77^{*} & 0,78^{*} & 0,74^{*} & 0,75^{*} \\ 0,74^{*} & 0,69^{*} & 0,71^{*} & 0,72^{*} & 0,78^{*} & 0,74^{*} & 0,73^{*} & 0,75^{*} & 0,69^{*} & 0,70^{*}\end{array}$

${ }^{1}$ Referenzkategorie: andere Ausbildung (d. h. berufliche Lehre und Berufsakademie) statt Hochschulstudium (d.h. Universität oder Fachhochschule)

* Mindestens $p \leq 0,05$

Quelle: Abiturientenbefragung in Sachsen im Juni 2000 - eigene Berechnungen

der sonstigen Fächer (10\%) zusammengefasst. Insgesamt stimmt die Verteilung der Studienfächer für die sächsischen Studienberechtigten weitgehend mit der Verteilung für die gesamte Bundesrepublik Deutschland überein (Heine u. Willich 2006, S. 22-24).

Der Zusammenhang zwischen sozialer Herkunft und Studienfachwahl unterstützt ebenfalls vorliegende Befunde (Modell 1 in Tabelle 5), wobei sich hier die allseits bekannten Zusammenhänge zwischen sozialer Herkunft und Studien- bzw. Ausbildungsentscheidung (siehe Becker u. Hecken 2007) widerspiegeln. ${ }^{12}$ Wie bereits im theoretischen Teil erläutert, reflektiert sie vor dem Hintergrund einer Berufswahl zwecks Statuserhalts aber auch die synchrone Entscheidung von Ausbildungsstätte und Inhalt einer Ausbildung. So gesehen unterschätzen unseres

\footnotetext{
${ }^{12}$ Mit dem von McFadden vorgeschlagenen Pseudo- $\mathrm{R}^{2}$ wird ein „,konservativeres" Pseudo- $\mathrm{R}^{2}$ wie das von Cox und Snell oder von Nagelkerke vorgeschlagene verwendet, die in der Regel drei bis vier Mal größer sind. Da dieses Pseudo- $\mathrm{R}^{2}$ nicht alle Eigenschaften wie die Maßzahl für statistisch erklärte Varianz einer linearen OLS-Regression besitzt, sollte es auch nicht ausschließlich als Güte für die Modellschätzung interpretiert werden. Sinnvoller ist es, das Pseudo- $\mathrm{R}^{2}$ eher für den Vergleich unterschiedlicher Modellspezifikationen, um Verbesserungen von Schätzungen abzulesen, als für die Gesamtgüte eines einzigen Modells heranzuziehen.
}

Erachtens empirische Studien, welche die Studienfachwahl von Studienanfängern untersuchen, die soziale Selektivität der Ausbildungsentscheidung. Gemessen an den Chancenverhältnissen für die nichttertiären Berufsausbildungen aber auch im Vergleich zu den anderen Studienfächern liegen für die naturwissenschaftlichen Disziplinen sowie für Architektur, Bauwesen, Mathematik und Information sowie für Ingenieurswissenschaften wie Elektrotechnik, Maschinenbau und Verfahrenstechnik die geringste soziale Selektivität vor, während für die Studienrichtungen Medizin und Jura die deutlichste Selektivität nach Klassenlage des Elternhauses festzustellen ist. Wie theoretisch erwartet, präferieren Studienberechtigte aus der oberen Dienstklasse im Kontrast zur nichttertiären Berufsausbildung fast fünf Mal eher als Arbeiterkinder oder Abiturienten aus den Mittelschichten das Studium der Medizin und fast vier Mal eher das Studium der Rechtswissenschaft (vgl. Reimer u. Pollak 2005). ${ }^{13}$ Auch für Studienrichtungen wie

\footnotetext{
${ }^{13}$ Diese an den Abstromprozenten aus der gesamten Risikomenge der Studienberechtigten orientierte Analyse verweist darauf, dass Berechnungen der sozialen Ungleichheit von Studienfachwahlen anhand von Zustromprozenten ebenso zu irreführenden Ergebnissen führt wie die ausschließliche Verwendung einer Stichprobe von Studierenden selbst.
} 
Geistes-, Wirtschafts- und Sozialwissenschaften oder Ingenieurswissenschaften, die im Allgemeinen als Fächer für Bildungsaufsteiger gelten, gibt es im Vergleich zu den anderen Berufsausbildungen eine deutliche herkunftsspezifische Differenzierung. Demnach entscheiden sich gegenüber den Abiturienten aus den Arbeiter- und Mittelschichten vor allem Studienberechtigte aus der oberen Dienstklasse für diese Studienfächer statt für eine nichttertiäre Ausbildung.

Insgesamt lässt sich festhalten, dass sich Studienberechtigte aus der oberen Dienstklasse im Vergleich zu Abiturienten aus den anderen Sozialschichten eher für Medizin und Jura als für andere nichttertiäre Ausbildungsalternativen entscheiden. Für die beiden Studienfächer Medizin und Jura ist dies vor dem Hintergrund des theoretisch vorausgesetzten Motivs für Statuserhalt, des geringen Kostendrucks, der geringen soziokulturellen Distanz zu diesen Studienfächern und den primären Herkunftseffekten zu erwarten. Ob diese Annahme empirisch zutreffend ist, soll überprüft werden, indem die Komponenten und Mechanismen des Entscheidungsprozesses statistisch kontrolliert werden (Modell 2 in Tabelle 5). Wenn die Einflüsse der sozialen Herkunft auf die Studienfachwahl durch die theoretisch abgeleiteten Mechanismen und Prozesse auspartialisiert werden, dann hat es sich als tragfähig erwiesen.

Werden zusätzlich zu der selbstbewerteten Schulleistung $P$ und dem erwarteten Studienerfolg $p$ als Indikatoren für primäre Herkunftseffekte die Erwartungswerte für den beruflichen Nutzen $U$, den Betrag des Statusverlusts $-S V$, die Wahrscheinlichkeit eines Statusverlusts $c$ bei „Fehlentscheidungen“, die unmittelbaren Studienkosten $C$ sowie die soziokulturelle Distanz $S D$ als Indikatoren für die sekundären Herkunftseffekte in die Modellschätzung einbezogen, dann werden für alle Studienfächer die Effekte der sozialen Herkunft insignifikant. ${ }^{14}$ Gemessen am Pseudo- ${ }^{2}$-Wert hat sich diese Modellspezifikation signifikant von 0,004 auf 0,089 verbessert und sagt mehr über die Verteilung der Ausbildungsalternativen zwischen den Abiturienten aus, als wenn nur deren soziale Herkunft kontrolliert wird. Damit hat sich unsere hauptsächliche Hypothese empirisch bestätigt. Der Zusammenhang zwischen Studienfachwahl und sozialer Herkunft lässt sich bei Kontrolle der selbstbewerteten Schulperformanz und - daraus abgeleitet - der subjektiv antizipierten Zulassungsrestriktionen weitgehend auf die theoretisch abgeleiteten Determinanten der Ausbildungs-

\footnotetext{
${ }^{14}$ Bislang wurde die Signifikanz der Herkunftseffekte immer in Bezug zur Referenzkategorie gesetzt. Ohne Kontrolle der die Studienfachwahl determinierenden Faktoren sind signifikante Klassenunterschiede zwischen den Studienfächern ersichtlich. Diese werden bei Kontrolle der primären und sekundären Herkunftseffekte insignifikant, sodass der Zusammenhang zwischen sozialer Herkunft und Studienfachwahl bzw. die signifikante Klassenunterschiede zwischen den Studienfächern auf klassenspezifische Kosten-Nutzen-Kalküle, Statuserhaltungsmotive, Erfolgswahrscheinlichkeiten und soziokulturelle Distanzen zurückzuführen sind (vgl. Tabelle 4).
}

entscheidung bzw. auf das Zusammenspiel von primären und sekundären Herkunftseffekten vor dem Hintergrund des institutionellen Bildungsangebots und den erworbenen Anrechten für bestimmte Studienfächer zurückführen. ${ }^{15}$

Ergänzend ist zu erwähnen, dass - wie zuvor theoretisch vermutet - für die Entscheidung für Medizin und Jura starke Selektionseffekte der schulischen Performanz und erwarteter Ausbildungskosten sowie vergleichsweise ausgeprägte Statuserhaltungsmotive vorliegen. Neben Zulassungsbeschränkungen angesichts unzureichender Schulperformanz dürften Kostenerwartungen dazu führen, dass Studienberechtigte mit Medizin als beabsichtigtem Studienfach überproportional häufig auf ein Medizinstudium verzichten oder auch ebenso häufig ein anderes Studienfach beginnen oder überdurchschnittlich zu einem Studienfachwechsel im Studienverlauf neigen (Heine et al. 2007, S. 62-64).

\subsection{Weiterführender Modelltest}

Im theoretischen Teil war auf die Rolle der Endogenität des vorherigen Bildungsverlaufs, gemessen an der Wahl von Leistungskursen in der gymnasialen Oberstufe, und die sich daraus ergebende oder verstärkende Interessen- und Motivationslage sowie die darauf basierende Studienfachwahl eingegangen worden. Vor diesem Hintergrund soll der Frage nachgegangen werden, ob inhaltliche Interessen, die mehr oder weniger herkunftsbedingt sind, die soziale Selektivität von Studienfachwahlen erklären können. Zunächst wird der Zusammenhang zwischen sozialer Herkunft und Leistungskurse beleuchtet (Tabelle 6). Aus datentechnischen Gründen können lediglich die drei Erhebungen aus den Jahren 2002, 2004 und 2006 berücksichtigt werden. Von allen erhobenen Leistungskursen weisen Englisch, Physik, Geschichte und Sport die größte Selektivität auf. Während Englisch eher von Abiturienten aus den mittleren und höheren Sozialschichten, Physik und Geschichte eher von Abiturienten aus der oberen Dienstklasse belegt werden, wird Sport eher von den Arbeiterkindern als Leistungskurs gewählt. Aber für Sport ist der Effekt der sozialen Herkunft insignifikant.

Nunmehr wird der Effekt der von den Befragten belegten Leistungskurse auf ihre Studienfachwahl betrachtet (Modell 1 in Tabelle 7). Um die Zahl der zu schätzenden Koeffizienten möglichst gering zu halten, sind die Leistungskurse zu Kategorien wie Sprachen, Naturwissen-

\footnotetext{
${ }^{15}$ Der statistisch signifikante Herkunftseffekt für die Wahl von Ingenieurswissenschaften oder von Mathematik bzw. Informatik als Studienrichtung kann mittels der Werterwartungstheorie nicht sinnvoll interpretiert werden. $\mathrm{Ob}$ dieses Faktum, dass Abiturienten aus den Mittelschichten eine geringe Neigung haben, diese Studienfächer zu studieren, ein periodenspezifischer Zufallsbefund ist, haben wir überprüft. Wenn wir lediglich die Befragungen ab 2002 berïcksichtigen, sind für alle Ausbildungsalternativen die Effekte für die Klassenlage des Elternhauses insignifikant.
} 
Tabelle 6 Leistungskurswahl nach sozialer Herkunft - Multinomiale Logit-Regression (odds ratios)

\begin{tabular}{lccccccccccc}
\hline & Deutsch & Mathe & Englisch & Biologie & Chemie & Physik & Geschichte & Kunst & Musik & Sport \\
\hline Arbeiterklassen & 1 & 1 & 1 & 1 & 1 & 1 & 1 & 1 & 1 & 2,23 \\
Mittelschichten & 1,14 & 1,10 & $1,32^{*}$ & 1,09 & 0,90 & $1,38^{*}$ & 1,23 & 0,99 & 0,57 & 1 \\
Obere Dienstklasse & 1,18 & 1,21 & $1,40^{*}$ & 1,03 & 0,82 & $1,79^{*}$ & $1,68^{*}$ & 1,28 & 1,04 & 1 \\
& & & & & & & & & & \\
Pseudo-R & & & & & & & & & \\
(McFadden) & 0,001 & 0,001 & 0,003 & 0,000 & 0,001 & 0,005 & 0,004 & 0,001 & 0,007 & 0,011 \\
$\begin{array}{l}\text { Fallzahl } \\
\text { Prozentuale }\end{array}$ & 4244 & 4244 & 4244 & 4244 & 4244 & 4244 & 4244 & 4244 & 4244 & 4244 \\
Anteile & $48,4 \%$ & $30,3 \%$ & $26,0 \%$ & $20,7 \%$ & $3,8 \%$ & $11,0 \%$ & $13,0 \%$ & $1,2 \%$ & $0,5 \%$ & $0,3 \%$ \\
\hline
\end{tabular}

* Mindestens $p \leq 0,05$

Quelle: Abiturientenbefragung in Sachsen 2002-2006 - eigene Berechnungen

Tabelle 7 Studienfachwahl nach sozialer Herkunft, schulische Schwerpunkte und Determinanten der Studienfachwahl ${ }^{1}-$ Multinomiale Logit-Regression (odds ratios)

\begin{tabular}{|c|c|c|c|c|c|c|c|c|c|c|}
\hline & $\begin{array}{l}\text { Andere } \\
\text { Fächer }\end{array}$ & Medizin & Jura & ET/MB & SoWi & WiWi & $\begin{array}{l}\text { Natur- } \\
\text { wissen- } \\
\text { schaften }\end{array}$ & $\begin{array}{l}\text { Geistes- } \\
\text { wissen- } \\
\text { schaften }\end{array}$ & $\begin{array}{c}\text { Architek- } \\
\text { tur/Bau- } \\
\text { wesen }\end{array}$ & $\begin{array}{c}\text { Mathe/ } \\
\text { Informatik }\end{array}$ \\
\hline \multicolumn{11}{|c|}{ Modell 1: Pseudo- $\mathrm{R}^{2}($ McFadden $)=0,062$} \\
\hline \multicolumn{11}{|l|}{ Soziale Herkunft } \\
\hline Arbeiterklassen & 1 & 1 & 1 & 1 & 1 & 1 & 1 & 1 & 1 & 1 \\
\hline Mittelschichten & $1,34^{*}$ & $1,69^{*}$ & 1,63 & 1,13 & $1,42^{*}$ & $1,65^{*}$ & $1,38^{*}$ & $1,39^{*}$ & 1,27 & 1,19 \\
\hline Obere Dienstklasse & $2,07^{*}$ & $3,43^{*}$ & $3,91^{*}$ & $1,84^{*}$ & $2,02^{*}$ & $2,12^{*}$ & 1,31 & $1,70^{*}$ & $2,15^{*}$ & 1,22 \\
\hline \multicolumn{11}{|l|}{ Leistungskurse } \\
\hline Sprachen & $0,70^{*}$ & $1,77^{*}$ & 2,12 & $0,23^{*}$ & 1,23 & $0,65^{*}$ & $0,67^{*}$ & $2,90^{*}$ & $0,34^{*}$ & 0,74 \\
\hline Naturwissenschaften & 1,14 & $3,20^{*}$ & 0,63 & 1,32 & 0,78 & $0,54^{*}$ & $3,39^{*}$ & $0,49^{*}$ & 0,65 & 1,01 \\
\hline Mathematik & $1,57^{*}$ & $2,50^{*}$ & $2,66^{*}$ & $1,79^{*}$ & 0,90 & $1,58^{*}$ & $1,80^{*}$ & 1,23 & $1,70^{*}$ & $9,01^{*}$ \\
\hline Geschichte & 1,31 & 0,80 & 1,97 & 0,66 & $1,48^{*}$ & 1,37 & 0,69 & $1,79^{*}$ & 1,04 & 0,57 \\
\hline$\%$ Anteile $(\mathrm{N}=4244)$ & $9,4 \%$ & $3,1 \%$ & $1,0 \%$ & $6,9 \%$ & $8,6 \%$ & $6,1 \%$ & $8,7 \%$ & $10,3 \%$ & $3,4 \%$ & $5,7 \%$ \\
\hline \multicolumn{11}{|c|}{ Modell 2: Pseudo- $\mathrm{R}^{2}($ McFadden $)=0,134$} \\
\hline \multicolumn{11}{|l|}{ Soziale Herkunft } \\
\hline Arbeiterklassen & 1 & 1 & 1 & 1 & 1 & 1 & 1 & 1 & 1 & 1 \\
\hline Mittelschichten & 1,03 & 1,03 & 0,77 & 0,80 & 1,11 & 1,09 & 1,03 & 0,96 & 0,83 & 0,85 \\
\hline Obere Dienstklasse & 1,18 & 1,18 & 1,12 & 0,93 & 1,44 & 1,08 & 0,85 & 0,99 & 1,13 & 0,73 \\
\hline \multicolumn{11}{|l|}{ Leistungskurse } \\
\hline Sprachen & $0,67^{*}$ & $0,67^{*}$ & 1,89 & $0,21^{*}$ & 1,23 & $0,53^{*}$ & $0,66^{*}$ & $2,91^{*}$ & $0,35^{*}$ & 0,70 \\
\hline Naturwissenschaften & 1,19 & 1,19 & 0,52 & 1,22 & 0,80 & $0,53^{*}$ & $3,66^{*}$ & $0,52^{*}$ & 0,68 & 1,01 \\
\hline Mathematik & 1,07 & 1,07 & 1,68 & 1,30 & 0,71 & 1,08 & 1,24 & 0,90 & 1,09 & $6,32^{*}$ \\
\hline Geschichte & 1,23 & 1,23 & 1,87 & 0,56 & 1,36 & 1,15 & 0,72 & $1,68^{*}$ & 1,02 & 0,58 \\
\hline \multicolumn{11}{|l|}{ Studienfachwahl } \\
\hline Schulleistung & $1,80^{*}$ & $1,80^{*}$ & $2,85^{*}$ & $1,57^{*}$ & $1,55^{*}$ & $2,22 *$ & $2,32^{*}$ & $1,77^{*}$ & 1,21 & $2,19^{*}$ \\
\hline Beruflicher Nutzen & $1,19^{*}$ & $1,19^{*}$ & $1,57^{*}$ & $1,47^{*}$ & $1,20^{*}$ & $1,43^{*}$ & $1,34^{*}$ & $1,12^{*}$ & 1,10 & $1,46^{*}$ \\
\hline$P$ (Statusverlust) & $1,77^{*}$ & $1,77^{*}$ & $1,98^{*}$ & $2,19^{*}$ & $1,77^{*}$ & $2,09^{*}$ & $1,69^{*}$ & $1,58^{*}$ & $1,61^{*}$ & $1,70^{*}$ \\
\hline Betrag des Statusverlusts & $1,54^{*}$ & $1,54^{*}$ & $1,85^{*}$ & $1,66^{*}$ & $1,51^{*}$ & $1,39^{*}$ & $1,55^{*}$ & $1,61^{*}$ & $1,62^{*}$ & $1,40^{*}$ \\
\hline Studienerfolg & $1,75^{*}$ & $1,75^{*}$ & 1,47 & $2,38^{*}$ & $2,08^{*}$ & $2,26^{*}$ & $1,90^{*}$ & $2,33^{*}$ & $2,61^{*}$ & $1,65^{*}$ \\
\hline Kosten für Studium & $0,82 *$ & $0,82^{*}$ & $0,59 *$ & $0,80^{*}$ & $0,86^{*}$ & $0,81^{*}$ & $0,79^{*}$ & $0,76^{*}$ & $0,74^{*}$ & $0,80^{*}$ \\
\hline Soziale Distanz & $0,77^{*}$ & $0,77^{*}$ & $0,67^{*}$ & $0,75^{*}$ & $0,82^{*}$ & $0,74^{*}$ & $0,82^{*}$ & $0,78^{*}$ & $0,76^{*}$ & $0,79^{*}$ \\
\hline
\end{tabular}

${ }^{1}$ Referenzkategorie: andere Ausbildung (d.h. berufliche Lehre und Berufsakademie) statt Hochschulstudium (d.h. Universität oder Fachhochschule)

${ }^{*}$ Mindestens $p \leq 0,05$

Quelle: Abiturientenbefragung in Sachsen 2002-2006 - eigene Berechnungen 
schaften, Mathematik und Geschichte zusammengefasst. Selten gewählte Leistungskurse wie Kunst, Musik und Sport, für die wegen leerer oder gering besetzter Zellen für die soziale Herkunft ohnehin Schätzprobleme auftraten, werden nicht weiter als eigenständige Kategorie berücksichtigt. Die vorliegenden Befunde sind wenig überraschend und höchst redundant, wenn die Mechanismen der Auswahl von Leistungskursen nicht kontrolliert werden kann. Dass beispielsweise Abiturienten, die Mathematik belegt haben, sich vor allem für Mathematik bzw. Informatik sowie auch eher für Natur-, Wirtschafts- oder Ingenieurswissenschaften als Studienfach entscheiden, ist ein ebenso wenig interessantes Ergebnis wie das Faktum, dass Abiturienten mit Geschichte als Leistungskurs vornehmlich Geistes- oder Sozialwissenschaften studieren werden. Dieser Befund kann auch als empirischer Beleg dafür gelten, dass die Wahl der Leistungskurse entsprechend des entscheidungsund handlungstheoretischen Ansatzes im Hinblick auf die Berufs- und Studienfachwahl strategisch - auch gemäß dem „Anlagesinn“ für die intergenerationale Statusreproduktion erfolgt und wenig mit eigenständiger intrinsischer Motivation zu tun hat. Vielmehr könnte es eine Folge des primären Herkunftseffektes sein. Erwähnenswert ist noch, dass Abiturienten mit Sprachen oder Mathematik als Leistungskurse entweder Jura oder Medizin studieren wollen, und diejenigen, die Naturwissenschaften als Leistungskurse belegt haben, nicht nur eher Naturwissenschaften studieren wollen, sondern auch Medizin. Informationsreich hingegen ist der Befund, dass bei Kontrolle der Leistungskurse der Zusammenhang von sozialer Herkunft und Studienfachwahl für die meisten Disziplinen weiterhin signifikant ist. Hiermit bestätigt sich die Behauptung, dass inhaltliche Neigungen, Interessen und Motivation zwar notwendige, aber keine hinreichenden Gründe für eine herkunftsbedingte Ausbildungs- und Berufswahl sind.

Führen wir die theoretisch begründeten Determinanten der Studienfachwahl in die Modellschätzungen ein, dann werden bei signifikant gestiegenem Pseudo- $\mathrm{R}^{2}$ die Herkunftseffekte erwartungsgemäß statistisch insignifikant (Modell 2 in Tabelle 7). ${ }^{16}$ Starke Nettoeffekte besuchter Leistungskurse bleiben allerdings weiterhin signifikant. Unklar ist jedoch, wie bereits angedeutet (und das kann mit den zur Verfügung stehenden Querschnittsdaten nicht geklärt werden), ob es sich hierbei um Selektionseffekte oder um Sozialisationseffekte handelt. Angesichts vorliegender Befunde ist zu vermuten, dass für Jura hauptsächlich

\footnotetext{
${ }^{16}$ Diese Modellierung in Tabelle 7 ist auch der empirische Beleg für unsere Vermutung, dass die singulären Effekte der sozialen Herkunft in Modell 2 in Tabelle 5 periodenspezifische Besonderheiten sind. Allerdings liefern diese marginalen Abweichungen einen Hinweis dafür, in Zukunft, wenn noch weitere Abiturientenbefragungen vorliegen, die historische Veränderungen des Arbeitsmarktgeschehens systematisch in Rechnung zu stellen (vgl. Becker 2000b).
}

Selektionseffekte dominieren, während bei der Entscheidung für Medizin auch Sozialisationseffekte durch die Schule bzw. den Leistungskurs vorliegen. Aufschluss über diese plausibel erscheinende Vermutung können nur noch Längsschnittdaten liefern, mit denen die Wahl der Leistungskurse und die Studienfächer modelliert werden kann.

\section{Zusammenfassung und Schlussfolgerung}

Ziel der vorliegenden Untersuchung war es, über die Beschreibung des Ausbildungsverhaltens von Abiturienten hinausgehend die Auswahl eines Studienfaches (einschließlich der zentralen Mechanismen und Prozesse) anhand eines entscheidungs- und handlungstheoretischen Erklärungsansatzes zu modellieren und somit die Schichtabhängigkeit der Studienfachwahl zu erklären. Um die Frage zu klären, warum es einen Zusammenhang zwischen sozialer Herkunft und Studienfachwahl gibt, ist in der theoretischen Rekonstruktion zunächst davon ausgegangen worden, dass die Studienfachwahl ein Spezialfall der Ausbildungsentscheidung und Berufswahl ist, die mehr oder weniger systematisch zwischen den Sozialschichten differiert. Der für die Studienfachwahl adaptierte strukturell-individualistische Erklärungsansatz stellt nicht nur darauf $a b$, dass diese Entscheidung eine zweckrationale Abwägung von Nutzen und Kosten bestimmter Studienrichtungen ist, sondern dass neben zweckrationalen Kalkulationen auch traditionale und wertrationale Bestimmungsgründe systematisch in dieses Erklärungsmodell integriert werden können. Auf diesem Wege konnte auch erklärt werden, warum es so wie beispielsweise von Bourdieu (1984) behauptet eine intergenerationale Transmission des Studienfachs gibt und warum hierbei höhere Sozialschichten gegenüber den Arbeiterklassen und unteren Mittelschichten deutlich im Vorteil sind. Es konnte theoretisch rekonstruiert werden, wie die Affinität von sozialer Herkunft und Studienfach zustande kommt und worin der ,Anlagesinn für die ,richtigen “ Ausbildungsgänge“" begründet liegt.

Die empirischen Analysen basieren auf Befragungen sächsischer Abiturientinnen und Abiturienten in Abschlussklassen allgemein bildender oder beruflicher Gymnasien sowie in Fachoberschulen. Die in den Jahren 2000, 2002, 2004 und 2006 durchgeführten Befragungen erfolgten jeweils kurz vor dem Erwerb der Hochschulberechtigung. Diese Datenbasis erlaubt es hinreichend gut, die soziale Selektivität von Ausbildungsentscheidungen im Allgemeinen und Studienfachwahlen im Besonderen unter Kontrolle sozialer Mechanismen $\mathrm{zu}$ beschreiben und $\mathrm{zu}$ modellieren.

In theoretisch sparsamer wie methodisch eleganter Weise kann empirisch nachgewiesen werden, dass die Studien- 
fachwahl in Abhängigkeit von der Schichtzugehörigkeit das Ergebnis von Entscheidungen ist, die vor allem durch das Motiv des Statuserhalts, den für bestimmte Studienfächer erwarteten Renditen, den erwarteten Kosten für bestimmte Studienrichtungen sowie den individuellen Leistungspotenzialen und den erwarteten Studienerfolgen strukturiert werden. Das von Esser (1999) oder von Erikson u. Jonsson (1996) oder von Breen u. Goldthorpe (1997) vorgeschlagene Modell der individuellen bzw. klassenspezifischen Bildungsentscheidungen lässt sich ohne Weiteres auf spezifische Ausbildungsentscheidungen und Berufswahlen übertragen. Auf diesem Wege erfahren die interessanten, aber unvollständigen kultursoziologischen Überlegungen von Bourdieu (1984) bzw. von Bourdieu u. Passeron (1978) eine entscheidungs- und handlungstheoretische Fundierung, die plausibel macht, warum gerade Studienfächer wie Medizin oder Jura von Abiturienten aus der oberen Dienstklasse gewählt werden und warum andere Studienfächer und Ausbildungen durch eine geringere soziale Selektivität gekennzeichnet sind.

Allerdings wurden auch Grenzen der verwendeten Datenbasis deutlich, die in der zukünftigen Forschung über eine entsprechende Datenerhebung ausgeräumt werden sollten. Zunächst müssen, um die intergenerationale Transmission von Studienfächern differenziert beschreiben und erklären zu können, für Studienberechtigte auch Informationen zu den Studienfächern, Berufsabschlüssen und beruflichen Tätigkeiten ihrer Eltern vorliegen. Des Weiteren benötigen wir Informationen zur intergenerationalen Transmission von Studienfächern über mindestens drei Generationen hinweg, um beurteilen zu können, dass bestimmte Familientraditionen und soziale Schließungen über diese Transmissionen verfolgt werden. Ebenso ist es notwendig, die Formierung von Ausbildungs- und Studienfachpräferenzen und Entscheidungsprozesse im Bildungsverlauf im Längsschnitt und in der Logik des Lebenslaufs von Schülerinnen und Schülern zu erheben. Erst auf diesem Weg ist es unseres Erachtens möglich, eine Fragestellung zu klären, die wir bewusst nicht berücksichtigt haben nämlich die Ursache für dauerhafte geschlechtsspezifische Segregationen bei den Studienfächern (Franzen et al. 2004). Denn bei eigenen, hier nicht präsentierten Analysen konnten die Geschlechterdisparitäten in der Studienfachwahl trotz Kontrolle von sozialer Herkunft und den Determinanten der Ausbildungsentscheidung (inklusive relevanter Kostenterme) nicht auspartialisiert werden (vgl. Böttcher et al. 1988, S. 14). Schließlich werden die vorliegenden Analysen nicht der Dynamik des Arbeitsmarktgeschehens gerecht. Es werden Daten benötigt, die für einen längeren Zeitraum die subjektive Einschätzung der Abiturienten über die Entwicklung des Arbeitsmarktes einfängt (vgl. Becker 2000a). Zumindest sind unsere Befunde für die erste Hälfte im ersten Jahrzehnt des 21. Jahrhunderts gültig.

\section{Kurzfassung}

Vorliegende Studien belegen eine persistente horizontale Ungleichheit von Studienfachwahlen nach sozialer Herkunft. So präferieren studienbereite Akademikerkinder vor allem Studienrichtungen wie etwa Medizin oder Jura, während Lehramtsstudiengänge oder Studienfächer wie Pädagogik oder Elektrotechnik besonders für Arbeiterkinder interessant zu sein scheinen. Es stellt sich die Frage, warum es einen Zusammenhang von sozialer Herkunft und Studienfachwahl dieser Art gibt. Abgesehen von Interessen und Neigungen werden das Motiv, den elterlichen Status zu erhalten, die quasi-ökonomische Abwägung von individuellen Leistungspotenzialen, beruflichen Renditen, des zu erwartenden Studienerfolgs und von anfallenden Kosten für ein Studienfach sowie die soziokulturelle Distanz zur tertiären Bildung als wichtige Mechanismen für die Entscheidung für ein bestimmtes Studienfach angesehen. Bei Kontrolle der Leistungskurse, die von den Abiturienten belegt wurden, wird diese Vermutung für sächsische Abiturienten bestätigt. Anhand dieser Determinanten der Studienfachwahl kann der Effekt sozialer Herkunft und damit - im Aggregat gesehen - die persistente soziale Ungleichheit von Studienfachwahlen nach sozialer Herkunft erklärt werden. Somit wird ein Beitrag zur Frage geliefert, warum gerade für prestigeträchtige Studienfächer wie Medizin oder Jura eine ausgeprägte intergenerationale Reproduktion gibt.

\section{Executive summary}

Recent studies provide empirical evidence on persistence of horizontal inequality of field of study depending on eligible individuals' social origin. For example, eligible children from the academic class prefer medicine or law while working-class children often study to become a teacher or prefer fields of study such as pedagogy or electrical engineering. However, the question arises why there is a correlation between social origin and choice of field of study. Apart from interest and affinity, the motive for status maintenance, the quasi-economic calculation of individual abilities, benefits (e.g. income or prestige), the anticipated likelihood of successful university training, the expected costs and the social distance to the tertiary education are important mechanisms for the choice of a field of study. Even if the advanced course attended by the high school graduates has been controlled, this assumption will be confirmed for high school graduates in Saxony. These determinants of the choice of field of study explains the effect of social origin and, therefore for the aggregate, the persistent social inequality of opportunities in the access to field of study. In this respect, our analysis contributes to the question about the reason for 
the intergenerational reproduction of field of study such as medicine or law.

Danksagung Für hilfreiche Kommentare danken wir Anna Etta Hecken, Walter Müller, David Reimer und besonders Reinhard Pollak sowie den anonymen Gutachtern.

\section{Literatur}

Arum, R., Gamoran, A., Shavit, Y.: More Inclusion Than Diversion: Expansion, Differentiation, and Market Structure in Higher Education. In: Shavit, Y., Arum, R., Gamoran, A. (eds.) Stratification in Higher Education, pp. 1-35. Stanford University Press, Stanford (2007)

Asmussen, J.: Leistungsmotivation, intrinsische Studienmotivation und Berufsorientierung als Determinanten der Studienfachwahl. In: Schmidt, U. (Hrsg.) Übergänge im Bildungssystem, S. 93-155. VS Verlag für Sozialwissenschaften, Wiesbaden (2006)

Becker, R.: Determinanten der Studierbereitschaft in Ostdeutschland. Eine empirische Anwendung der Humankapital- und Werterwartungstheorie am Beispiel sächsischer Abiturienten in den Jahren 1996 und 1998. Mitt. Arbeitsmarkt Berufsforsch. 33(2), 261-276 (2000a)

Becker, R.: Studierbereitschaft und Wahl von ingenieurwissenschaftlichen Studienfächern. Eine empirische Untersuchung sächsischer Abiturienten der Abschlussjahrgänge 1996,1998 und 2000. WZB-Discussion Paper FS I 00-210. WZB, Berlin (2000b)

Becker, R.: Educational expansion and persistent inequalities of education: Utilizing the subjective expected utility theory to explain the increasing participation rates in upper secondary school in the Federal Republic of Germany. Eur. Sociol. Rev. 19(1), 1-24 (2003)

Becker, R.: Warum bildungsferne Gruppen von der Universität fernbleiben und wie man sie für das Studium an der Universität gewinnen könnte. In: Krüger, H.-H., Rabe-Kleberg, U., Kramer, R.-T., Budde, J. (Hrsg.) Bildungsungleichheiten revisited. Bildung und soziale Ungleichheit vom Kindergarten bis zur Hochschule. VS Verlag für Sozialwissenschaften, Wiesbaden (2009) (im Druck)

Becker, R., Hecken, A.E.: Studium oder Berufsausbildung? Eine empirische Überprüfung der Modelle zur Erklärung von Bildungsentscheidungen von Esser sowie von Breen und Goldthorpe. Z. Soziol. 36(2), 100-117 (2007)

Becker, R., Hecken, A.E.: Warum werden Arbeiterkinder vom Studium an Universitäten abgelenkt? Eine empirische Überprüfung der „Ablenkungsthese“ von Müller und Pollak (2007) und ihrer Erweiterung durch Hillmert und Jacob (2003). Kölner Z. Soziol. Sozialpsychol. 60(1), 3-29 (2008)

Becker, R., Hecken, A.E.: Why are working-class children diverted from universities? An empirical assessment of the diversion thesis. Europ. Sociol. Rev. 25(2), 233-250 (2009a)

Becker, R., Hecken, A.E.: Higher education or vocational training? An empirical test of the rational action model of educational choices suggested by Breen and Goldthorpe (1997) and Esser (1999).Acta Sociol. 52(1), 25-45 (2009b)

Becker, R., Schömann, K.: Berufliche Weiterbildung und Einkommensdynamik. Eine Längsschnittstudie mit besonderer Berücksichtigung von Selektionsprozessen. Kölner Z. Soziol. Sozialpsychol. 48(3), 426-461 (1996)

Blossfeld, H.-P.: Changes in Educational Opportunities in the Federal Republic of Germany. A Longitudinal Study of Cohorts Born between 1916 and 1965. In: Shavit, Y., Blossfeld, H.-P. (Hrsg.) Persistent Inequality, pp. 51-74. Westview Press, Boulder (1993)
Böttcher, W., Holtappels, H.G., Rösner, E.: Abitur - und dann? Bildungswahlen von Schülern der gymnasialen Oberstufe. Pädagogik 40(5), 11-14 (1988)

Boudon, R.: Education, Opportunity, and Social Inequality. Wiley, New York (1974)

Bourdieu, P.: Ökonomisches Kapital, kulturelles Kapital, soziales Kapital. In: Kreckel, R. (Hrsg.) Soziale Ungleichheiten (Sonderband 2 der Sozialen Welt), S. 183-199. Otto Schwartz, Göttingen (1983)

Bourdieu, P.: Homo academicus. Suhrkamp, Frankfurt am Main (1984)

Bourdieu, P., Passeron, J.-C.: Die Illusion der Chancengleichheit. Klett, Stuttgart (1978)

Breen, R., Goldthorpe, J.H.: Explaining educational differentials. Towards a formal rational action theory. Ration. Soc. 9(3), 275-305 (1997)

Breen, R., Jonsson, J.O.: Analyzing educational careers: A multinomial transition model. Am. Sociol. Rev. 65(5), 754-772 (2000)

Brüderl, J.: Die Überprüfung von Rational-Choice-Modellen mit Umfragedaten. In: Diekmann, A., Voss, T. (Hrsg.) Rational-ChoiceTheorie in den Sozialwissenschaften. Anwendungen und Probleme, S. 163-180. Oldenbourg, München (2004)

Davies, S., Guppy, N.: Field of study, college selectivity, and student inequalities in higher education. Soc. Forces 75(4), 1417-1438 (1997)

Erikson, R., Jonsson, J.O.: Explaining Class Inequality in Education: The Swedish Test Case. In: Erikson, R., Jonsson, J.O. (Hrsg.) Can Education Be Equalized?, S. 1-63. Westview Press, Boulder (1996)

Esser, H.: Soziologie. Spezielle Grundlagen, Bd. 1, Situationslogik und Handeln. Campus, Frankfurt am Main (1999)

Esser, H.: Soziologie. Spezielle Grundlagen, Bd. 6, Sinn und Kultur. Campus, Frankfurt am Main (2001)

Franzen, A., Hecken, A.E., Kopp, C.: Bildungsexpansion und die geschlechtsspezifische Segregation an Schweizer Hochschulen. Soz. Welt 55(3), 317-335 (2004)

Friedrichs, J., Stolle, M., Engelbrecht, G.: Rational-Choice-Theorie: Probleme der Operationalisierung. Z. Soziol. 22(1), 2-15 (1993)

Georg, W.: Studienfachwahl: Soziale Reproduktion oder fachkulturelle Entscheidung. ZA-Inf. 57, 61-82 (2005)

Hansen, M. N.: Social and economic inequality in the educational career: Do the effects of social background characteristics decline? Europ. Sociol. Rev. 13(3), 305-321 (1997)

Heckman, J.J.: Sample selection bias as a specification error. Econometrica 47(1), 153-161 (1979)

Hedström, P., Swedberg, R.: Social mechanism. Acta Sociol. 39(3), 281-308 (1996)

Heine, C., Scheller, P.: Studium, Beruf und Werdegänge. HISKurzinformation A14/2005. HIS, Hannover (2005)

Heine, C., Spangenberg, H., Lörz, M.: Nachschulische Werdegänge studienberechtigter Schulabgänger/innen. Zweite Befragung der Studienberechtigten $2002 \quad 3^{1} / 2$ Jahre nach Schulabgang im Zeitvergleich. HIS Forum Hochschule Nr. F11/2007. HIS, Hannover (2007)

Heine, C., Spangenberg, H., Willich, J.: Studienberechtigte 2006 ein halbes Jahr nach Schulabschluss. HIS Forum Hochschule 4/2008. HIS, Hannover (2008)

Heine, C., Willich, J.: Studienberechtigte 2005: Übergang in Studium, Ausbildung und Beruf. HIS Forum Hochschule 6/2006. HIS, Hannover (2006)

Heublein, U., Sommer, D.: Studienanfänger 2000/2001: Fachinteresse und berufliche Möglichkeiten bestimmen die Studienfachwahl. HIS-Kurzinformation A2/2002. HIS, Hannover (2002)

Hillmert, S., Jacob, M.: Social inequality in higher education: Is vocational training a pathway leading to or away from university? Europ. Sociol. Rev. 19(3), 319-334 (2003) 
Kroneberg, C.: Die Definition der Situation und die variable Rationalität der Akteure. Z. Soziol. 34(5), 344-363 (2005)

Kroneberg, C.: Wertrationalität und das Modell der Frame-Selektion. Kölner Z. Soziol. Sozialpsychol. 59(2), 215-239 (2007)

Lindenberg, S.: Social production functions, deficits, and social revolutions. Ration. Soc. 1(1), 51-77 (1989)

Lischka, I., Wolter, A.: Studienaufnahme oder Studienverzicht? In: Lischka, I., Wolter, A. (Hrsg.) Hochschulzugang im Wandel? Entwicklungen, Reformperspektiven und Alternativen, S. 59-98. Beltz, Weinheim (2001)

Long, J.S.: Regression Models for Categorical and Limited Dependent Variables. Sage, Thousand Oaks (1997)

Lucas, S.R.: Effectively maintained inequality: Educational transitions and social background. Am. J. Sociol. 106(6), 16421690 (2001)

Maaz, K.: Soziale Herkunft und Hochschulzugang. Effekte institutioneller Öffnung im Bildungssystem. VS-Verlag für Sozialwissenschaften, Wiesbaden (2006)

Manski, C.F.: Measuring expectations. Econometrica 72(5), 1329$1376(2004)$

Mare, R.D.: Social background and school continuation decisions. J. Am. Statistical Assoc. 75(370), 295-305 (1980)

Mayer, K.U., Müller, W., Pollak, R.: Germany: Institutional Change and Inequalities of Access in Higher Education. In: Shavit, Y., Arum, R., Gamoran, A. (Hrsg.) Stratification in Higher Education, pp. 240-265. Stanford University Press, Stanford (2007)

Meulemann, H.: Bildung und Lebensplanung. Die Sozialbeziehung zwischen Elternhaus und Schule. Campus, Frankfurt am Main (1985)

Meulemann, H.: Soziologie von Anfang an. Westdeutscher Verlag, Wiesbaden (2001)

Müller, W.: Familie - Schule - Beruf. Soziale Mobilität und Prozesse der Statuszuweisung in der Bundesrepublik. Westdeutscher Verlag, Opladen (1975)

Müller, W.: Bildung und soziale Plazierung in Deutschland, England und Frankreich. Peisert, H., Zapf, W. (Hrsg.) Gesellschaft, Demokratie und Lebenschancen, S. 115-134. DVA, Frankfurt am Main (1994)

Müller, W., Brauns, H., Steinmann, S.: Expansion und Erträge tertiärer Bildung in Deutschland, Frankreich und im Vereinigten Königreich. Berl. J. Soziol. 12(1), 37-62 (2002)

Müller, W., Jacob, M.: Qualifications and the Returns to Training Across the Life Course. In: Mayer, K.U., Solga, H. (Hrsg.) Skill Formation, pp. 126-172. Cambridge University Press, Cambridge (2008)

Müller, W., Karle, W.: Social Selection in Educational Systems in Europe. Europ. Sociol. Rev. 9(1), 1-22 (1993)

Müller, W., Pollak, R.: Weshalb gibt es so wenige Arbeiterkinder in Deutschlands Universitäten? In: Becker, R., Lauterbach, W. (Hrsg.) Bildung als Privileg, 2., aktualis. Aufl., S. 303-342. VS Verlag für Sozialwissenschaften, Wiesbaden (2007)

Multrus, F.: Fachtraditionen bei Studierenden. Studienwahl und elterliche Fachrichtung. Hefte zur Bildungs- und Hochschulforschung 47. Arbeitsgruppe Hochschulforschung, Universität Konstanz (2006)

Opp, K.D., Friedrichs, J.: Brückenannahmen, Produktionsfunktionen und die Messung von Präferenzen. Kölner Z. Soziol. Sozialpsychol. 48(3), 546-559 (1996)

Opp, K.D., Friedrichs, J.: Rational behavior in everyday situations. Europ. Sociol. Rev. 18(4), 401-415 (2002)
Reimer, D., Pollak, R.: The Impact of Social Origin on the Transition to Tertiary Education in West Germany 1983 and 1999. MZESWorking paper No. 85. MZES, Mannheim (2005)

Reimer, D., Pollak, R.: Educational Expansion and Its Consequences for Vertical and Horizontal Inequalities in Access to Higher Education in West Germany. Europ. Sociol. Rev. (2009) (Advance access publiziert am 11. Juni 2009)

Schölling, M.: Soziale Herkunft, Lebensstil und Studienfachwahl: eine Typologie. Lang, Frankfurt am Main (2005)

Urban, D.: Logit-Analyse. Statistische Verfahren zur Analyse von Modellen mit qualitativen Response-Variablen. Fischer, Stuttgart (1993)

Van de Werfhorst, H.G., de Graaf, N.D., Kraaykamp, G.: Intergenerational Resemblance in Field of Study in the Netherlands. Europ. Sociol. Rev. 17(3), 275-293 (2001)

Weber, M.: Wirtschaft und Gesellschaft. Mohr Siebeck, Tübingen (1980)

Wegener, B.: Kritik des Prestiges. Westdeutscher Verlag, Opladen (1988)

Windolf, P.: Fachkultur und Studienfachwahl. Ergebnisse einer Befragung von Studienanfängern. Kölner Z. Soziol. Sozialpsychol. 42(1), 76-98 (1990)

Wolter, A., Lenz, K., Laskowski, R.: Studierbereitschaft bleibt auf hohem Niveau. Die Studien- und Berufswahl von Studienberechtigten des Abschlussjahrgangs 2006 in Sachsen. Eine empirische Untersuchung des Sächsischen Staatsministeriums für Kultus in Zusammenarbeit mit der Technischen Universität Dresden (Abschlussbericht). TU Dresden und SMK, Dresden (2006)

Rolf Becker, Studium der Soziologie und Politikwissenschaft an der Universität Mannheim, 1987 Abschluss als Diplom-Soziologe, 1991 Promotion und 1999 Habilitation. Seit 2004 Direktor der Abteilung Bildungssoziologie und Professor für Bildungssoziologie an der Universität Bern.

Forschungsfelder: Bildungssoziologie, Sozialstrukturanalyse, Lebensverlaufsforschung, Methoden der empirischen Sozialforschung und angewandte Statistik, Arbeitsmarkt- und Mobilitätsforschung, empirische Wahlforschung, Familien- und Generationenbeziehungen E-Mail: rolf.becker@edu.unibe.ch

Sigrid Haunberger, Studium der Soziologie, Arbeits- und Organisationspsychologie und Statistik an der Ludwig-Maximilians-Universität München, 2004 Abschluss als Diplom-Soziologin. Seit 2004 wissenschaftliche Assistentin an der Universität Bern in der Abteilung Bildungssoziologie.

Forschungsfelder: Methoden und Techniken empirischer Sozialforschung (insbesondere Interviewereffekte und Unit-Nonresponse), Bildungssoziologie, Soziologie der Kindheit

E-Mail: sigrid.haunberger@edu.unibe.ch

Frank Schubert, Studium der Soziologie an der Universität Mannheim, 2004 Abschluss als Diplom-Sozialwissenschaftler. Seit 2004 wissenschaftlicher Mitarbeiter an der Universität Bern in der Abteilung Bildungssoziologie.

Forschungsfelder: International vergleichende Bildungsforschung, Übertritt vom Bildungssystem in den Arbeitsmarkt, Mobilitätsforschung, Methoden der empirischen Sozialforschung und Datenanalyseverfahren.

E-Mail: frank.schubert@edu.unibe.ch 